\title{
Palladium-Catalyzed Cyclization/Carboalkoxylation of
}

\author{
Alkenyl Indoles
}

Cong Liu and Ross A. Widenhoefer*

P. M. Gross Chemical Laboratory

Duke University

Durham, NC 27708-0346

rwidenho@chem.duke.edu

\section{Supporting Information}

Experimental procedures, analytical and spectroscopic data for new compounds and copies of NMR spectra (30 pages). 


\section{Experimental}

General Methods. NMR spectra were obtained on a Varian spectrometer operating at 400 $\mathrm{MHz}$ for ${ }^{1} \mathrm{H} \mathrm{NMR}$ and $100 \mathrm{MHz}$ for ${ }^{13} \mathrm{C} \mathrm{NMR}$ in $\mathrm{CDCl}_{3}$ unless noted otherwise. IR spectra were obtained on a Bomen MB-100 FT-IR spectrometer. Gas chromatography was performed on a HewlettParkard 5890 gas chromatography equipped with a $25 \mathrm{~m}$ polydimethylsiloxane capillary column. Flash column chromatography was performed employing 200-400 mesh silica gel (EM). Thin layer chromatography (TLC) was performed on silica gel $60 \mathrm{~F}_{254}$. Elemental analyses were performed by Complete Analysis Laboratories (Parsippany, NJ) or Robertson Microlit Laboratories (Madison, NJ).

Anhydrous methanol (Aldrich) was uses as received. Anhydrous THF (Fischer) was distilled from sodium benzophenone ketyl under nitrogen prior to use. $\operatorname{PdCl}_{2}\left(\mathrm{MeCN}_{2}(\mathbf{2}), \mathrm{PdCl}_{2}\left(\mathrm{PPh}_{3}\right)_{2}\right.$, $\left[\mathrm{PtCl}_{2}\left(\mathrm{CH}_{2}=\mathrm{CH}_{2}\right)\right]_{2}, \mathrm{Pd}(\mathrm{OAc})_{2},($ Aldrich $), \mathrm{CuCl}_{2}$ (Strem), methyl isobutyrate, trans-1-bromo-2-pentene, cis-1-bromo-2-pentene, ethyl 6-heptenoate, 4-fluoro-2-methylaniline (Acros), and methyl 5-hexenoate (TCI) were used as received. $\quad \mathrm{N}$-Trimethylsilyl-o-toluidine (S1), ${ }^{1} \mathrm{~N}$-trimethylsilyl-4-fluoro-o-toluidine, ${ }^{1}$ 1, ${ }^{2}$ 2-(4-pentenyl)-1H-indole (S2, Table 1, entry 1), ${ }^{2}$ 5-methoxy-1-methyl-2-(4-pentenyl)-1H-indole (Table 1, entry 2), 2-(1,1-dimethyl-4-pentenyl)-1-methyl-1H-indole (Table 1, entry 4), ${ }^{2}$ 2-(2,2dicarbomethoxy-4-pentenyl)-1-methyl-1H-indole (Table 1, entry 5), ${ }^{2} \mathrm{~N}$-allyl- $\mathrm{N}$-methyl-1-methyl-1Hindole-2-carboxamide (Table 1, entry 6), ${ }^{3}$ 1-methyl-2-(4-methyl-4-pentenyl)-1H-indole (Table 1 , entry 7), ${ }^{2}$ cis-2-(2,2-dicarbomethoxy-4-heptenyl)-1-methyl-1H-indole (Table $\quad 1, \quad$ entry 8$){ }^{2}$ dimethyl 2-(2cyclohexenyl)-2-(1-methyl-1H-indol-2-ylmethyl)malonate (Table 1 , entry 9$),{ }^{2} 1$-methyl-2-(3-butenyl)-1Hindole (Table 1 , entry 10), ${ }^{2}$ 3-(1-methyl-1H-indol-3-yl)-propionaldehyde, ${ }^{4} 3$-(4-pentenyl)-1H-indole (Table 1 , entry 15$)$ were synthesized employing published procedures. ${ }^{5}$ 


\section{Alkenyl Indoles}

5-Fluoro-2-(4-pentenyl)-1H-indole (Table 1, entry 3). 5-Fluoro-2-(4-pentenyl)-1H-indole was isolated in $29 \%$ yield as a pale yellow oil from the the reaction of $N$-trimethylsilyl-4-fluoro- $O$ toluidine and methyl 5-hexenoate employing a procedure similar to that used to synthesize S2. ${ }^{2}$ TLC (hexanes- $\left.\mathrm{CH}_{2} \mathrm{Cl}_{2}=1: 1\right): R_{f}=0.50 .{ }^{1} \mathrm{H} \mathrm{NMR}: \delta 7.84(\mathrm{~s}, 1 \mathrm{H}), 7.20-7.17(\mathrm{~m}, 2 \mathrm{H}), 6.87(\mathrm{dt}, J=2.6,9.2$ $\mathrm{Hz}, 1 \mathrm{H}), 6.23-6.22(\mathrm{~m}, 1 \mathrm{H}), 5.85(\mathrm{tdd}, J=6.6,10.4,17.2 \mathrm{~Hz}, 1 \mathrm{H}), 5.11-5.03(\mathrm{~m}, 2 \mathrm{H}), 2.76(\mathrm{t}, J=7.2$ $\mathrm{Hz}, 2 \mathrm{H}$ ), $2.17(\mathrm{q}, J=7.2 \mathrm{~Hz}, 2 \mathrm{H}), 1.83$ (quintet, $J=7.2 \mathrm{~Hz}, 2 \mathrm{H}) .{ }^{13} \mathrm{C}\left\{{ }^{1} \mathrm{H}\right\} \mathrm{NMR}: \delta 158.2\left(\mathrm{~d},{ }^{1} J_{\mathrm{CF}}=\right.$ $232 \mathrm{~Hz}), 141.8,138.4,132.6,129.6\left(\mathrm{~d},{ }^{3} J_{\mathrm{CF}}=10 \mathrm{~Hz}\right), 115.6,111.0\left(\mathrm{~d},{ }^{3} J_{\mathrm{CF}}=10 \mathrm{~Hz}\right), 109.3\left(\mathrm{~d},{ }^{2} J_{\mathrm{CF}}=26\right.$ $\mathrm{Hz}), 105.0\left(\mathrm{~d},{ }^{2} J_{\mathrm{CF}}=24 \mathrm{~Hz}\right), 100.2,33.5,28.5,27.9$. IR (neat, $\left.\mathrm{cm}^{-1}\right): 3415,2932,1585,1485,1453,1416$, 1313, 1167, 1105, 953. Anal. calcd (found) for $\mathrm{C}_{13} \mathrm{H}_{14} \mathrm{FN}: \mathrm{C}, 76.82$ (76.80); H, 6.94 (6.78).

trans-2-(1,1-Dimethyl-3-hexenyl)-1H-indole (Table 1, entry 12). Methyl isobutyrate (3.06 g, $30.0 \mathrm{mmol}$ ) was added to a solution of LDA [generated from $n$-BuLi and diisopropylamine] in THF at -78 ${ }^{\circ} \mathrm{C}$. The resulting solution was stirred for $30 \mathrm{~min}$ at $-78{ }^{\circ} \mathrm{C}$ and treated with trans-1-bromo-2-pentene (4.00 g, $30.0 \mathrm{mmol})$. The resulting mixture was warmed to room temperature, stirred for $4 \mathrm{~h}$, and quenched with saturated aqueous $\mathrm{NH}_{4} \mathrm{Cl}$. The layers were separated and the aqueous layer was extracted with ether $(3 \times 50 \mathrm{~mL})$. The combined organic extracts were dried $\left(\mathrm{MgSO}_{4}\right)$ and concentrated under vacuum. Column chromatography of the residue $\left(\mathrm{SiO}_{2} ;\right.$ hexanes-EtOAc $\left.=10: 1\right)$ gave methyl trans-2,2-dimethyl-4-heptenoate (S3) (3.82 g, 75\%) as a colorless oil. A solution of $n$-BuLi in hexanes (2.5 M, 15.0 mL, $37.5 \mathrm{mmol})$ was added dropwise to a solution of $\mathbf{S 1}(2.69 \mathrm{~g}, 15.0 \mathrm{mmol})$ in hexanes (50 $\mathrm{mL}$ ) at $0{ }^{\circ} \mathrm{C}$. The resulting orange solution was refluxed for $6 \mathrm{~h}$, cooled to $-78{ }^{\circ} \mathrm{C}$, and treated with a solution of S3 $(2.55 \mathrm{~g}, 15.0 \mathrm{mmol})$ in THF $(10 \mathrm{~mL})$. The resulting mixture was warmed to room temperature, stirred for $1 \mathrm{~h}$, and quenched with brine. The layers were separated and the aqueous layer was extracted with ether $(2 \times 30 \mathrm{~mL})$. The combined organic extracts were dried $\left(\mathrm{MgSO}_{4}\right)$ and 
concentrated under vacuum. Column chromatography of the residue $\left(\mathrm{SiO}_{2} ;\right.$ hexanes- $\left.-\mathrm{CH}_{2} \mathrm{Cl}_{2}=10: 1\right)$ gave trans-2-(1,1-dimethyl-3-hexenyl)-1H-indole (2.42 g, 71\%) as a pale yellow oil.

For S3: TLC (hexanes-EtOAc $=10: 1): R_{f}=0.61 .{ }^{1} \mathrm{H}$ NMR: $\delta 5.46(\operatorname{ttd}, J=1.2,6.2,15.2 \mathrm{~Hz}, 1$ H), $5.29(\mathrm{ttd}, J=1.6,7.2,14.8 \mathrm{~Hz}, 1 \mathrm{H}), 3.64(\mathrm{~s}, 3 \mathrm{H}), 2.17(\mathrm{dd}, J=0.8,7.2 \mathrm{~Hz}, 2 \mathrm{H}), 1.98$ (quint, $J=7.2$ $\mathrm{Hz}, 2 \mathrm{H}), 1.13$ (s, $6 \mathrm{H}), 0.94(\mathrm{t}, J=7.6 \mathrm{~Hz}, 3 \mathrm{H}) .{ }^{13} \mathrm{C}\left\{{ }^{1} \mathrm{H}\right\}$ NMR: $\delta \quad 178.5,136.1,124.7,51.9,43.9,43.0$, 26.0, 25.1, 14.3. IR (neat, $\mathrm{cm}^{-1}$ ): 2968, 1733, 1433, 1200, 1135, 990, 867, 713. Anal. calcd (found) for $\mathrm{C}_{10} \mathrm{H}_{18} \mathrm{O}_{2}: \mathrm{C}, 70.55$ (70.61); H, $10.66(10.83)$.

For trans-2-(1,1-dimethyl-3-hexenyl)-1 $H$-indole: TLC (hexanes- $\left.\mathrm{CH}_{2} \mathrm{Cl}_{2}=1: 1\right): \quad R_{f}=0.65$. ${ }^{1} \mathrm{H}$ NMR: $\delta 8.00(\mathrm{~s}, 1 \mathrm{H}), 7.58(\mathrm{dd}, J=0.8,8.2 \mathrm{~Hz}, 1 \mathrm{H}), 7.34(\mathrm{dd}, J=0.8,7.6 \mathrm{~Hz}, 1 \mathrm{H}), 7.19-7.09$ (m, 2 H), $6.30(\mathrm{~m}, 1 \mathrm{H}), 5.53(\mathrm{ttd}, J=1.4,7.2,15.0 \mathrm{~Hz}, 1 \mathrm{H}), 5.32(\mathrm{ttd}, J=1.2,7.0,15.2 \mathrm{~Hz}, 1 \mathrm{H}), 2.35$ (dd, $J=$ $1.2,7.2 \mathrm{~Hz}, 2 \mathrm{H}), 2.02$ (quint, $J=7.8 \mathrm{~Hz}, 2 \mathrm{H}), 1.38(\mathrm{~s}, 6 \mathrm{H}), 0.98(\mathrm{t}, J=7.6 \mathrm{~Hz}, 3 \mathrm{H}) .{ }^{13} \mathrm{C}\left\{{ }^{1} \mathrm{H}\right\}$ NMR: $\delta$ 147.9, 136.1, 136.0, 128.8, 125.4, 121.3, 120.3, 119.8, 110.7, 98.3, 46.8, 35.4, 28.0, 26.0, 14.3. IR (neat, $\mathrm{cm}^{-1}$ ): $3424,2962,2929,1455,1294,969,784,749,693$. Anal. calcd (found) for $\mathrm{C}_{16} \mathrm{H}_{21} \mathrm{~N}: \mathrm{C}, 84.53$ (84.37); H, 9.31 (9.22).

cis-2-(1,1-Dimethyl-3-hexenyl)-1H-indole (Table 1, entry 11). cis-2,2-Dimethyl-4-heptenoate (S4) was isolated in 53\% yield as a colorless oil from the the reaction of methyl isobutyrate and cis-1bromo-2-pentene employing a procedure similar to that used to synthesize S3. cis-2-(1,1-Dimethyl-3hexenyl)- $1 H$-indole was isolated in $66 \%$ yield as a pale yellow oil from the reaction of $\mathbf{S 1}$ and $\mathbf{S 4}$ employing a procedure similar to that used to synthesize $\mathbf{S 2}$.

For S4: TLC (hexanes-EtOAc $=10: 1): R_{f}=0.60 .{ }^{1} \mathrm{H}$ NMR: $\delta 5.46(\mathrm{ttd}, J=1.6,7.2,10.8 \mathrm{~Hz}, 1$ H), $5.25(\operatorname{ttd}, J=1.6,7.0,10.8 \mathrm{~Hz}, 1 \mathrm{H}), 3.65(\mathrm{~s}, 3 \mathrm{H}), 2.26(\mathrm{~d}, J=7.6 \mathrm{~Hz}, 2 \mathrm{H}), 2.03$ (quint, $J=7.2 \mathrm{~Hz}, 2$ $\mathrm{H}), 1.17(\mathrm{~s}, 6 \mathrm{H}), 0.94(\mathrm{t}, J=7.6 \mathrm{~Hz}, 3 \mathrm{H}) .{ }^{13} \mathrm{C}\left\{{ }^{1} \mathrm{H}\right\}$ NMR: $\delta$ 178.5, 134.7, 124.3, 52.0, 42.8, 38.0, 25.1, 20.9, 14.5. IR (neat, $\mathrm{cm}^{-1}$ ): 2966, 2874, 1734, 1433, 1198, 1142, 867. Anal. calcd (found) for $\mathrm{C}_{10} \mathrm{H}_{18} \mathrm{O}_{2}$ : C, 70.55 (70.50); H, $10.66(10.83)$. 
For cis-2-(1,1-dimethyl-3-hexenyl)-1 $\boldsymbol{H}$-indole: $\mathrm{TLC}$ (hexanes- $\mathrm{CH}_{2} \mathrm{Cl}_{2}=1: 1$ ): $\quad \boldsymbol{R}_{f}=0.67 . \quad{ }^{1} \mathrm{H}$ NMR: $\delta 8.00(\mathrm{~s}, 1 \mathrm{H}), 7.57(\mathrm{dd}, J=0.8,8.0 \mathrm{~Hz}, 1 \mathrm{H}), 7.32(\mathrm{dd}, J=0.8,7.6 \mathrm{~Hz}, 1 \mathrm{H}), 7.17-7.07(\mathrm{~m}, 2 \mathrm{H})$, 6.30-6.29 (m, $1 \mathrm{H}), 5.48(\mathrm{ttd}, J=1.6,7.2,10.8 \mathrm{~Hz}, 1 \mathrm{H}), 5.30(\mathrm{ttd}, J=1.6,7.0,10.8 \mathrm{~Hz}, 1 \mathrm{H}), 2.41(\mathrm{~d}, J=$ $8.2 \mathrm{~Hz}, 2 \mathrm{H}$ ), 2.03 (quint, $J=7.6 \mathrm{~Hz}, 2 \mathrm{H}), 1.40(\mathrm{~s}, 6 \mathrm{H}), 0.94(\mathrm{t}, J=7.6 \mathrm{~Hz}, 3 \mathrm{H}) .{ }^{13} \mathrm{C}\left\{{ }^{1} \mathrm{H}\right\} \mathrm{NMR}: \delta$ 147.6, 136.0, 134.5, 1286, 125.1, 121.3, 120.2, 119.8, 110.6, 98.3, 40.8, 35.5, 28.0, 20.9, 14.3. IR (neat, $\mathrm{cm}^{-1}$ ): 3424, 2963, 1460, 1296, 784. Anal. calcd (found) for $\mathrm{C}_{16} \mathrm{H}_{21} \mathrm{~N}: \mathrm{C}, 84.53$ (84.40); H, 9.31 (9.48).

2-(5-Hexenyl)-1-methylindole (Table 1, entry 13). 2-(5-Hexenyl)-1H-indole (S5) was isolated in 50\% yield as a pale yellow oil from the reaction of $\mathbf{S 1}$ and ethyl 6-heptenoate employing a procedure similar to that used to synthesize S2. 2-(5-Hexenyl)-1-methyl-1H-indole was isolated in $74 \%$ yield as a colorless oil from the reaction of $\mathbf{S 5}$ and methyl iodide employing a procedure similar to that used to synthesize $\mathbf{1}$.

For S5: TLC (hexanes- $\left.\mathrm{CH}_{2} \mathrm{Cl}_{2}=1: 1\right): R_{f}=0.56 .{ }^{1} \mathrm{H} \mathrm{NMR}: \delta 7.79(\mathrm{~s}, 1 \mathrm{H}), 7.59-7.57(\mathrm{~m}, 1 \mathrm{H})$, 7.32-7.29 (m, 1 H), 7.09-7.03 (m, 2 H), 6.29-6.27 (m, 1 H), 5.85 (tdd, $J=6.6,10.4,17.2 \mathrm{~Hz}, \mathrm{H}), 5.10-5.00$ (m, $2 \mathrm{H}$ ), 2.77 (t, $J=7.6 \mathrm{~Hz}, 2 \mathrm{H}$ ), 2.15 (q, $J=7.6 \mathrm{~Hz}, 2 \mathrm{H}$ ), 1.77 (quint, $J=7.6 \mathrm{~Hz}, 2 \mathrm{H}$ ), 1.54 (quint, $J=$ $7.6 \mathrm{~Hz}, 2 \mathrm{H}) .{ }^{13} \mathrm{C}\left\{{ }^{1} \mathrm{H}\right\}$ NMR: $\delta$ 147.1, 138.9, 136.1, 129.1, 121.2, 120.0, 119.9, 115.0, 110.6, 99.8, 33.8, 28.9, 28.8, 28.4. IR (neat, $\left.\mathrm{cm}^{-1}\right): 3404,2930,1550,1457,1414,1287,909,779,748$. Anal. calcd (found) for $\mathrm{C}_{14} \mathrm{H}_{17} \mathrm{~N}$ : C, 84.37 (84.25); H, 8.60 (8.42).

For 2-(5-Hexenyl)-1-methylindole: $\mathrm{TLC}$ (hexanes- $\left.\mathrm{CH}_{2} \mathrm{Cl}_{2}=5: 1\right): \quad R_{f}=0.38 . \quad{ }^{1} \mathrm{H}$ NMR: $\delta$ 7.62-7.60 (m, $1 \mathrm{H}), 7.34-7.32(\mathrm{~m}, 1 \mathrm{H}), 7.25-7.21(\mathrm{~m}, 2 \mathrm{H}), 6.33-6.31(\mathrm{~m}, 1 \mathrm{H}), 5.90$ (tdd, $J=6.6,10.4$, $17.2 \mathrm{~Hz}, 1 \mathrm{H}), 5.14-5.03(\mathrm{~m}, 2 \mathrm{H}), 3.70(\mathrm{~s}, 3 \mathrm{H}), 2.80(\mathrm{t}, J=7.6 \mathrm{~Hz}, 2 \mathrm{H}), 2.20(\mathrm{q}, J=7.6 \mathrm{~Hz}, 2 \mathrm{H}), 1.81$ (quint, $J=7.6 \mathrm{~Hz}, 2 \mathrm{H}$ ), 1.61 (quint, $J=7.6 \mathrm{~Hz}, 2 \mathrm{H}$ ). ${ }^{13} \mathrm{C}\left\{{ }^{1} \mathrm{H}\right\}$ NMR: $\delta \quad 141.5,138.9,137.6,128.2$, 120.8, 120.1, 119.5, 115.0, 109.0, 99.0, 33.9, 29.7, 28.9, 28.4, 27.0. IR (neat, $\mathrm{cm}^{-1}$ ): 2931, 1546, 1468, 909, 770, 746. Anal. calcd (found) for $\mathrm{C}_{15} \mathrm{H}_{19} \mathrm{~N}$ : C, 84.46 (84.22); H, 8.98 (8.84). 
3-(3-Butenyl)-1-methylindole (Table 1, entry 14). $n$-BuLi (2.5 M solution in hexanes, 4.4 $\mathrm{mL}, 11.0 \mathrm{mmol})$ was added dropwise to a mixture of $\mathrm{Ph}_{3} \mathrm{PCH}_{3} \mathrm{Br}(3.93 \mathrm{~g}, 11.0 \mathrm{mmol})$ in $\mathrm{THF}(50 \mathrm{~mL})$ at 0 ${ }^{\circ} \mathrm{C}$. The resulting mixture was stirred at $0{ }^{\circ} \mathrm{C}$ for $1 \mathrm{~h}$ and treated with a solution of 3-(1-methyl-1H-indol3-yl)-propionaldehyde $(0.79 \mathrm{~g}, 4.2 \mathrm{mmol})$ in THF $(10 \mathrm{~mL})$. The resulting mixture was warmed to room temperature, stirred for $2.5 \mathrm{~h}$, and quenched with saturated aqueous $\mathrm{NH}_{4} \mathrm{Cl}$. The aqueous layer was extracted with ether $(3 \times 30 \mathrm{~mL})$ and the layers were separated. The combined organic extracts were dried $\left(\mathrm{MgSO}_{4}\right)$ and concentrated under vacuum. Column chromatography of the residue $\left(\mathrm{SiO}_{2}\right.$; hexanes-EtOAc $=5: 1)$ gave 3-(3-butenyl)-1-methylindole $(0.70 \mathrm{~g}, 90 \%)$ as a pale yellow oil. TLC (hexanes-EtOAc $=2: 1): R_{f}=0.71 .{ }^{1} \mathrm{H}$ NMR: $\delta$ 7.70-7.68 $(\mathrm{m}, 1 \mathrm{H}), 7.42-7.28(\mathrm{~m}, 2 \mathrm{H}), 7.19(\mathrm{dt}, J=0.8$, $7.6 \mathrm{~Hz}, 1 \mathrm{H}), 6.90(\mathrm{~s}, 1 \mathrm{H}), 6.03(\mathrm{tdd}, J=6.6,10.4,17.2 \mathrm{~Hz}, 1 \mathrm{H}), 5.21-5.07$ (m, $2 \mathrm{H}), 3.79(\mathrm{~s}, 3 \mathrm{H}), 2.94(\mathrm{t}$, $J=7.2 \mathrm{~Hz}, 2 \mathrm{H}), 2.58(\mathrm{q}, J=7.2 \mathrm{~Hz}, 2 \mathrm{H}) .{ }^{13} \mathrm{C}\left\{{ }^{1} \mathrm{H}\right\} \mathrm{NMR}: \delta \quad 139.2,137.3,128.2,126.4,121.8,119.3$, 118.8, 114.9, 109.4, 34.9, 32.8, 25.0. IR (neat, $\mathrm{cm}^{-1}$ ): 3060, 2913, 1640, 1470, 1376, 1322, 910. Anal. calcd (found) for $\mathrm{C}_{13} \mathrm{H}_{15} \mathrm{~N}$ : C, 84.28 (84.01); H, 8.16 (8.06). 


\section{Cyclization/Carboalkoxyation of Alkenyl Indoles}

\section{Methanol as solvent}

Table S1. Effect of $\operatorname{Pd}(\mathrm{II})$ source and termial oxidant on the palladium-catalyzed cyclization/carboalkoxylation of $\mathbf{1}$.
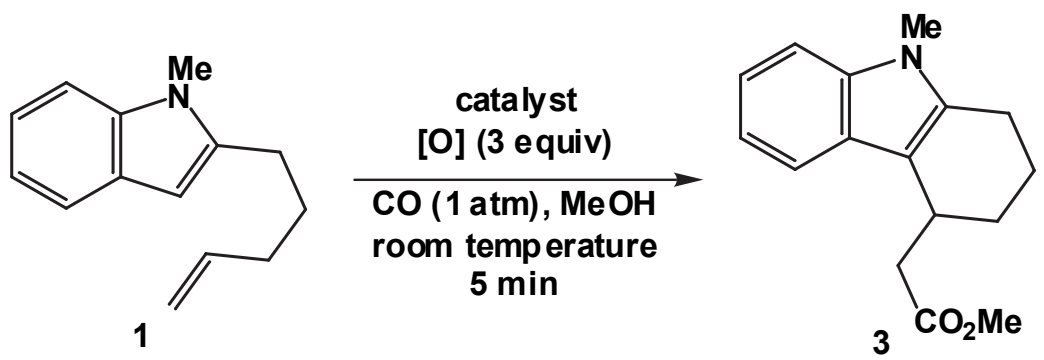

\begin{tabular}{lccc}
\hline catalyst & cat load (mol\%) & {$[\mathrm{O}]$} & yield (\%) \\
\hline 2 & 10 & $\mathrm{CuCl}_{2}$ & 94 \\
$\mathrm{PdCl}_{2}\left(\mathrm{PPh}_{3}\right)_{2}$ & 10 & $\mathrm{CuCl}_{2}$ & 15 \\
$\mathrm{Pd}(\mathrm{OAC})_{2}$ & 10 & $\mathrm{CuCl}_{2}$ & 18 \\
{$\left[\mathrm{PtCl}_{2}\left(\mathrm{CH}_{2}=\mathrm{CH}_{2}\right)\right]_{2}$} & 5 & $\mathrm{CuCl}_{2}$ & $<1$ \\
2 & 10 & $\mathrm{BQ}$ & $<1$ \\
2 & 5 & $\mathrm{CuCl}_{2}$ & 85 \\
2 & 2 & $\mathrm{CuCl}_{2}$ & 31 \\
\hline
\end{tabular}

${ }^{\mathrm{a}}$ As determined by GC analysis of the crude reaction mixture.

Methyl (9-methyl-2,3,4,9-tetrahydro-1H-carbazol-4-yl)-acetate (3). Methanol (4 mL) and a solution of $1(99 \mathrm{mg}, 0.50 \mathrm{mmol})$ in methanol $(1.0 \mathrm{~mL})$ were added sequentially to a flask containing $\mathrm{PdCl}_{2}(\mathrm{MeCN})_{2}(2)(6.5 \mathrm{mg}, 0.025 \mathrm{mmol})$ and $\mathrm{CuCl}_{2}(200 \mathrm{mg}, 1.5 \mathrm{mmol})$ that had been twice evacuated (12 $\mathrm{mmHg})$ and backfilled with $\mathrm{CO}$ (1 atm, balloon). The resulting suspension was stirred for $30 \mathrm{~min}$ and concentrated under vacuum. Column chromatography of the residue $\left(\mathrm{SiO}_{2}\right.$; hexanes-EtOAc $\left.=5: 1\right)$ gave $3(106 \mathrm{mg}, 83 \%)$ as a pale yellow oil. TLC (hexanes-EtOAc $=5: 1): R_{f}=0.27 .{ }^{1} \mathrm{H}$ NMR: $\delta 7.57(\mathrm{~d}, J=$ $8.4 \mathrm{~Hz}, 1 \mathrm{H}), 7.29(\mathrm{~d}, J=8.2 \mathrm{~Hz}, 1 \mathrm{H}), 7.20(\mathrm{t}, J=7.6 \mathrm{~Hz}, 1 \mathrm{H}), 7.11(\mathrm{t}, J=7.6 \mathrm{~Hz}, 1 \mathrm{H}), 3.76(\mathrm{~s}, 3 \mathrm{H})$, 
$3.63(\mathrm{~s}, 3 \mathrm{H}), 3.63-3.58(\mathrm{~m}, 1 \mathrm{H}), 3.04(\mathrm{dd}, J=4.0,15.2 \mathrm{~Hz}, 1 \mathrm{H}), 2.79-2.64(\mathrm{~m}, 2 \mathrm{H}), 2.47(\mathrm{dd}, J=10.4$, $14.8 \mathrm{~Hz}, 1 \mathrm{H}), 2.02-1.92(\mathrm{~m}, 3 \mathrm{H}), 1.87-1.79(\mathrm{~m}, 1 \mathrm{H}) .{ }^{13} \mathrm{C}\left\{{ }^{1} \mathrm{H}\right\}$ NMR: $\delta$ 173.9, 137.2, 136.4, 126.5, $120.9,119.1,118.3,111.5,109.0,51.9,40.2,29.4,29.0,28.6,22.4,19.5$. IR (neat, $\mathrm{cm}^{-1}$ ): 2934, 1731, $1470,1434,1416,1379,1281,1166,1080,1014,739$. Anal. calcd (found) for $\mathrm{C}_{16} \mathrm{H}_{19} \mathrm{NO}_{2}$ : $\mathrm{C}, 74.68$ (74.95); H, 7.44 (7.47).

All polycyclic indoles in Table 1 were synthesized employing a procedure similar to that used to synthesize 3 .

Methyl (2,3,4,9-tetrahydro-1H-carbazol-4-yl)-acetate (S6, Table 1, entry 1). ${ }^{6}$ Pale yellow oil, 83\%. TLC (hexanes-EtOAc $=2: 1): R_{f}=0.62 .{ }^{1} \mathrm{H}$ NMR: $\delta 7.82(\mathrm{~s}, 1 \mathrm{H}), 7.56(\mathrm{~d}, J=7.6 \mathrm{~Hz}, 1 \mathrm{H})$, $7.24(\mathrm{dd}, J=0.8,7.6 \mathrm{~Hz}, 1 \mathrm{H}), 7.17-7.09(\mathrm{~m}, 2 \mathrm{H}), 3.76(\mathrm{~s}, 3 \mathrm{H}), 3.60-3.57$ (m, $1 \mathrm{H}), 3.04(\mathrm{dd}, J=4.0$, $15.2 \mathrm{~Hz}, 1 \mathrm{H}), 2.70-2.64(\mathrm{~m}, 2 \mathrm{H}), 2.47(\mathrm{dd}, J=10.4,14.8 \mathrm{~Hz}, 1 \mathrm{H}), 2.00-1.79(\mathrm{~m}, 4 \mathrm{H}) .{ }^{13} \mathrm{C}\left\{{ }^{1} \mathrm{H}\right\} \mathrm{NMR}$ : $\delta 174.1,136.0,134.9,127.0,121.2,119.4,118.3,112.1,110.9,51.9,39.9,29.3,28.7,23.4,19.6$. IR (neat, $\left.\mathrm{cm}^{-1}\right): 3399,2932,1733,1464,1434,1326,1283,1168,1072,1012,741$

Methyl (6-methoxy-9-methyl-2,3,4,9-tetrahydro-1H-carbazol-4-yl)-acetate (Table 1 , entry 2). Pale yellow oil, $86 \%$. TLC (hexanes-EtOAc $=2: 1): \quad R_{f}=0.57 .{ }^{1} \mathrm{H}$ NMR: $\delta 7.16(\mathrm{~d}, J=9.2 \mathrm{~Hz}, 1$ H), $7.00(\mathrm{~d}, J=2.4 \mathrm{~Hz}, 1 \mathrm{H}), 6.84(\mathrm{dd}, J=2.4,8.4 \mathrm{~Hz}, 1 \mathrm{H}), 3.88(\mathrm{~s}, 3 \mathrm{H}), 3.75(\mathrm{~s}, 3 \mathrm{H}), 3.58(\mathrm{~s}, 3 \mathrm{H})$, 3.58-3.54 (m, $1 \mathrm{H}), 2.97(\mathrm{dd}, J=4.0,15.2 \mathrm{~Hz}, 1 \mathrm{H}), 2.76-2.60(\mathrm{~m}, 2 \mathrm{H}), 2.44(\mathrm{dd}, J=10.4,14.8 \mathrm{~Hz}, 1 \mathrm{H})$, 1.98-1.74 (m, $4 \mathrm{H}) .{ }^{13} \mathrm{C}\left\{{ }^{1} \mathrm{H}\right\} \mathrm{NMR}: \delta$ 173.9, 153.9, 137.0, 132.4, 126.6, 111.1, 110.4, 109.5, 100.9, 56.3, 51.7, 39.8, 29.2, 28.5, 22.4, 19.3. IR (neat, $\left.\mathrm{cm}^{-1}\right):$ 2934, 1732, 1621, 1579, 1484, 1415, 1281, 1254, 1223, 1153, 1080, 793. Anal. calcd (found) for $\mathrm{C}_{17} \mathrm{H}_{21} \mathrm{NO}_{3}$ : C, 71.06 (71.16); H, 7.37 (7.45).

Methyl (6-fluoro-2,3,4,9-tetrahydro-1H-carbazol-4-yl)-acetate (Table 1, entry 3). Colorless oil, 74\%. TLC (hexanes-EtOAc = 2:1): $R_{f}=0.45 .{ }^{1} \mathrm{H}$ NMR (Figure S1): $\delta 7.78(\mathrm{~s}, 1 \mathrm{H}), 7.19-7.14(\mathrm{~m}$, $2 \mathrm{H}), 6.85(\mathrm{dt}, J=2.6,9.0 \mathrm{~Hz}, 1 \mathrm{H}), 3.73(\mathrm{~s}, 3 \mathrm{H}), 3.52-3.46(\mathrm{~m}, 1 \mathrm{H}), 2.92(\mathrm{dd}, J=4.0,15.2 \mathrm{~Hz}, 1 \mathrm{H})$, 2.720-2.66 (m, $2 \mathrm{H}), 2.42(\mathrm{dd}, J=10.4,14.8 \mathrm{~Hz}, 1 \mathrm{H}), 1.97-1.75(\mathrm{~m}, 4 \mathrm{H}) .{ }^{13} \mathrm{C}\left\{{ }^{1} \mathrm{H}\right\}$ NMR (Figure S2): $\delta$ $173.8,158.0\left(\mathrm{~d},{ }^{1} J_{\mathrm{CF}}=233 \mathrm{~Hz}\right), 136.9,132.5,127.5\left(\mathrm{~d},{ }^{3} J_{\mathrm{CF}}=10 \mathrm{~Hz}\right), 112.8,111.3\left(\mathrm{~d},{ }^{3} J_{\mathrm{CF}}=10 \mathrm{~Hz}\right), 109.3$ 
$\left(\mathrm{d},{ }^{2} J_{\mathrm{CF}}=26 \mathrm{~Hz}\right), 103.6\left(\mathrm{~d},{ }^{2} J_{\mathrm{CF}}=24 \mathrm{~Hz}\right), 51.9,39.8,29.2,28.8,23.6,19.6$. IR (neat, $\left.\mathrm{cm}^{-1}\right): 3409,2936$, 1713, 1583, 1484, 1453, 1317, 1290, 1169, 1128, 797. HRMS calcd (found) for $\mathrm{C}_{15} \mathrm{H}_{16} \mathrm{FNO}_{2}\left(\mathrm{M}^{+}\right)$: 261.1165 (261.1159).

Methyl (1,1,9-trimethyl-2,3,4,9-tetrahydro-1H-carbazol-4-yl)-acetate (Table 1, entry 4). Pale yellow oil, 84\%. TLC (hexanes-EtOAc $=2: 1): R_{f}=0.74 .{ }^{1} \mathrm{H}$ NMR: $\delta 7.60(\mathrm{~d}, J=7.6 \mathrm{~Hz}, 1 \mathrm{H})$, $7.32(\mathrm{~d}, J=8.0 \mathrm{~Hz}, 1 \mathrm{H}), 7.25$ (t, $J=7.6 \mathrm{~Hz}, 1 \mathrm{H}), 7.15(\mathrm{t}, J=7.4 \mathrm{~Hz}, 1 \mathrm{H}), 3.86(\mathrm{~s}, 3 \mathrm{H}), 3.80(\mathrm{~s}, 3 \mathrm{H})$, 3.65-3.60 (m, $1 \mathrm{H}), 3.02(\mathrm{dd}, J=3.6,15.2 \mathrm{~Hz}, 1 \mathrm{H}), 2.53(\mathrm{dd}, J=11.2,15.2 \mathrm{~Hz}, 1 \mathrm{H}), 2.10$ (ddt, $J=2.4$, 5.2, $12.8 \mathrm{~Hz}, 1 \mathrm{H}), 1.95(\mathrm{dt}, J=2.0,13.6 \mathrm{~Hz}, 1 \mathrm{H}), 1.83-1.78(\mathrm{~m}, 1 \mathrm{H}), 1.67$ (ddd, $J=2.4,5.6,13.2 \mathrm{~Hz}, 1$ $\mathrm{H}), 1.54(\mathrm{~s}, 3 \mathrm{H}), 1.43(\mathrm{~s}, 3 \mathrm{H}) .{ }^{13} \mathrm{C}\left\{{ }^{1} \mathrm{H}\right\}$ NMR: $\delta 173.9,142.7,137.9,126.1,121.4,119.2,118.5,111.5$, 109.0, 51.8, 39.7, 38.4, 32.5, 32.2, 29.7, 29.2, 27.7, 25.1. IR (neat, $\mathrm{cm}^{-1}$ ): 2932, 1747, 1727, 1470, 1285, 1165, 1015, 742. Anal. calcd (found) for $\mathrm{C}_{18} \mathrm{H}_{23} \mathrm{NO}_{2}$ : C, 75.76 (75.60); $\mathrm{H}, 8.12$ (8.12).

Methyl (2,2 dicarbomethoxy-9-methyl-2,3,4,9-tetrahydro-1H-carbazol-4-yl)-acetate (Table 1, entry 5). Pale yellow oil, 87\%. TLC (hexanes-EtOAc $=2: 1): R_{f}=0.43 .{ }^{1} \mathrm{H}$ NMR: $\delta 7.51(\mathrm{~d}, J=8.0$ Hz, $1 \mathrm{H}), 7.29$ (d, $J=8.4 \mathrm{~Hz}, 1 \mathrm{H}), 7.19(\mathrm{t}, J=7.8 \mathrm{~Hz}, 1 \mathrm{H}), 7.08(\mathrm{t}, J=7.8 \mathrm{~Hz}, 1 \mathrm{H}), 3.80$ (s, $3 \mathrm{H}), 3.75$ (s, $3 \mathrm{H}), 3.69$ (s, $3 \mathrm{H}), 3.67$ (s, $3 \mathrm{H}), 3.70-3.63(\mathrm{~m}, 1 \mathrm{H}), 3.45$ (d, $J=16.4 \mathrm{~Hz}, 1 \mathrm{H}), 3.31$ (dd, $J=3.6,15.8 \mathrm{~Hz}$, $1 \mathrm{H}), 3.20(\mathrm{~d}, J=16.0 \mathrm{~Hz}, 1 \mathrm{H}), 2.86(\mathrm{dd}, J=6.0,13.6 \mathrm{~Hz}, 1 \mathrm{H}), 2.32(\mathrm{dd}, J=10.4,15.8 \mathrm{~Hz}, 1 \mathrm{H}), 2.05$ $(\mathrm{dd}, J=8.8,13.6 \mathrm{~Hz}, 1 \mathrm{H}) .{ }^{13} \mathrm{C}\left\{{ }^{1} \mathrm{H}\right\}$ NMR: $\delta 173.1,172.2,170.9,137.8,133.4,125.9,121.3,119.3$, 119.0, 109.6, 109.3, 54.4, 53.2, 51.9, 51.7, 40.3, 35.4, 29.5, 28.6, 28.4. IR (neat, $\mathrm{cm}^{-1}$ ): $2953,1747,1722$, 1085, 1018, 738. Anal. calcd (found) for $\mathrm{C}_{20} \mathrm{H}_{23} \mathrm{NO}_{6}$ : C, 64.33 (64.48); H, 6.21 (6.29).

Methyl (2,9-dimethyl-1-oxo-2,3,4,9-tetrahydro-1 $H$ - $\beta$-carbolin-4-yl)-acetate (Table 1 , entry 6). Colorless oil, 91\%. TLC (hexanes-EtOAc $=10: 1): R_{f}=0.21 .{ }^{1} \mathrm{H}$ NMR: $\delta 7.61(\mathrm{td}, J=0.8,8.0 \mathrm{~Hz}$, $1 \mathrm{H}), 7.37-7.30(\mathrm{~m}, 2 \mathrm{H}), 7.14(\mathrm{dt}, J=1.0,8.0 \mathrm{~Hz}, 1 \mathrm{H}), 4.11(\mathrm{~s}, 3 \mathrm{H}), 3.94(\mathrm{dd}, J=4.8,12.8 \mathrm{~Hz}, 1 \mathrm{H})$, $3.68(\mathrm{~s}, 3 \mathrm{H}$ ), 3.67-3.63 (X portion of ABX, partially obscured, $1 \mathrm{H}$ ), 3.46 (dd, $J=6.4,12.8 \mathrm{~Hz}, 1 \mathrm{H}$ ), 3.12 $(\mathrm{s}, 3 \mathrm{H}), 2.68,2.66\left(\mathrm{AB}\right.$ portion of $\left.\mathrm{ABX}, J_{\mathrm{AB}}=15.2 \mathrm{~Hz}, 2 \mathrm{H}\right) .{ }^{13} \mathrm{C}\left\{{ }^{1} \mathrm{H}\right\}$ NMR: $\delta$ 172.8, 161.8, 139.4, 126.3, 124.9, 123.2, 120.8, 120.5, 120.3, 110.6, 54.3, 52.1, 37.3, 34.7, 31.5, 29.1. IR (neat, $\mathrm{cm}^{-1}$ ): 2948, 
1727, 1649, 1493, 1440, 1248, 1165, 1082, 742. Anal. calcd (found) for $\mathrm{C}_{16} \mathrm{H}_{18} \mathrm{~N}_{2} \mathrm{O}_{3}$ : C, 67.12 (66.98); H, 6.34 (6.38).

Methyl (4,9-dimethyl-2,3,4,9-tetrahydro-1H-carbazol-4-yl)-acetate (Table 1, entry 7). Pale yellow solid, 87\%. $\mathrm{mp} 85-87{ }^{\circ} \mathrm{C}$. TLC (hexanes-EtOAc $\left.=2: 1\right): R_{f}=0.72 .{ }^{1} \mathrm{H}$ NMR: $\delta \quad 7.68(\mathrm{~d}, J=8.0$ $\mathrm{Hz}, 1 \mathrm{H}), 7.28(\mathrm{~d}, J=8.0 \mathrm{~Hz}, 1 \mathrm{H}), 7.18(\mathrm{t}, J=7.6 \mathrm{~Hz}, 1 \mathrm{H}), 7.09(\mathrm{t}, J=7.6 \mathrm{~Hz}, 1 \mathrm{H}), 3.66(\mathrm{~s}, 3 \mathrm{H}), 3.61(\mathrm{~s}$, $3 \mathrm{H}), 3.04(\mathrm{~d}, J=13.6 \mathrm{~Hz}, 1 \mathrm{H}), 2.70(\mathrm{dd}, J=5.6,12.0 \mathrm{~Hz}, 2 \mathrm{H}), 2.65(\mathrm{~d}, J=13.6 \mathrm{~Hz}, 1 \mathrm{H}), 2.16-2.08(\mathrm{~m}$, $1 \mathrm{H}), 1.97$ (quint, $J=6.0 \mathrm{~Hz}, 2 \mathrm{H}), 1.75-1.69(\mathrm{~m}, 1 \mathrm{H}), 1.60(\mathrm{~s}, 3 \mathrm{H}) .{ }^{13} \mathrm{C}\left\{{ }^{1} \mathrm{H}\right\} \mathrm{NMR}: \delta$ 173.0, 137.2, 135.6, 125.7, 120.6, 119.8, 118.8, 116.0, 109.1, 51.5, 45.6, 36.8, 35.0, 29.3, 27.2, 22.6, 19.7. IR (neat, $\mathrm{cm}^{-}$ $\left.{ }^{1}\right): 2933,1727,1469,1433,1412,1373,1314,1092,1018$. Anal. calcd (found) for $\mathrm{C}_{17} \mathrm{H}_{21} \mathrm{NO}_{2}: \mathrm{C}, 75.25$ (75.03); H, 7.80 (7.55).

\section{2,2-Dicarbomethoxy-4-(1-carbomethoxypropyl)-9-methyl-1,3,4,9-tetrahydrocarbazole}

(Table 1, entry 8). White solid, 62\%. mp 174-176 ${ }^{\circ} \mathrm{C}$. $\quad$ TLC (hexanes-EtOAc $=2: 1$ ): $R_{f}=0.41 . \quad{ }^{1} \mathrm{H}$ NMR: $\delta 7.57(\mathrm{~d}, J=8.0 \mathrm{~Hz}, 1 \mathrm{H}), 7.28(\mathrm{~d}, J=8.0 \mathrm{~Hz}, 1 \mathrm{H}), 7.18(\mathrm{t}, J=7.8 \mathrm{~Hz}, 1 \mathrm{H}), 7.07(\mathrm{t}, J=7.6 \mathrm{~Hz}$, $1 \mathrm{H}), 3.93-3.91(\mathrm{~m}, 1 \mathrm{H}), 3.80(\mathrm{~s}, 3 \mathrm{H}), 3.79(\mathrm{~s}, 3 \mathrm{H}), 3.66(\mathrm{~s}, 3 \mathrm{H}), 3.620(\mathrm{~s}, 3 \mathrm{H}), 3.52(\mathrm{~d}, J=16.0 \mathrm{~Hz}, 1$ H), 3.35 (br d, $J=11.2 \mathrm{~Hz}, 1 \mathrm{H}), 3.07$ (d, $J=15.8 \mathrm{~Hz}, 1 \mathrm{H}), 2.45$ (dd, $J=5.0,13.0 \mathrm{~Hz}, 1 \mathrm{H}), 2.02$ (dd, $J=$ 11.2, $14.8 \mathrm{~Hz}, 1 \mathrm{H}), 1.69-1.60(\mathrm{~m}, 1 \mathrm{H}), 1.06-0.97(\mathrm{~m}, 1 \mathrm{H}) . \quad{ }^{13} \mathrm{C}\left\{{ }^{1} \mathrm{H}\right\}$ NMR: $\delta \quad 175.6,172.3,170.7$, $137.9,134.4,126.1,121.2,119.3,109.3,108.8,55.0,53.4,53.1,52.0,49.6,34.6,30.9,29.6,28.3,18.4$, 13.5. IR (neat, $\mathrm{cm}^{-1}$ ): 2952, 1730, 1469, 1437, 1048, 737. Anal. calcd (found) for $\mathrm{C}_{22} \mathrm{H}_{27} \mathrm{NO}_{6}$ : $\mathrm{C}, 65.82$ (65.81); H, 6.78 (6.70).

\section{1,5,5-Tricarbomethoxy-7-methyl-2,3,4a,6,7,11c-hexahydro- $1 H, 4 H$-benzo[c]carbazole}

(Table 1, entry 9). White solid, 45\%. mp 174-176 ${ }^{\circ} \mathrm{C}$. $\quad$ TLC (hexanes-EtOAc $=2: 1$ ): $\quad R_{f}=0.41 . \quad{ }^{1} \mathrm{H}$ NMR: $\delta 7.55(\mathrm{~d}, J=8.0 \mathrm{~Hz}, 1 \mathrm{H}), 7.30(\mathrm{~d}, J=8.4 \mathrm{~Hz}, 1 \mathrm{H}), 7.17(\mathrm{t}, J=7.6 \mathrm{~Hz}, 1 \mathrm{H}), 7.04(\mathrm{t}, J=7.8 \mathrm{~Hz}$, $1 \mathrm{H}), 3.93-3.91(\mathrm{~m}, 1 \mathrm{H}), 3.90-3.88(\mathrm{~m}, 1 \mathrm{H}), 3.84(\mathrm{~s}, 3 \mathrm{H}), 3.80(\mathrm{~s}, 3 \mathrm{H}), 3.71(\mathrm{~s}, 3 \mathrm{H}), 3.70$ (s, $3 \mathrm{H}), 3.55$ $(\mathrm{d}, J=17.6 \mathrm{~Hz}, 1 \mathrm{H}), 3.11(\mathrm{dd}, J=2.6,17.4 \mathrm{~Hz}, 1 \mathrm{H}), 3.08(\mathrm{td}, J=3.8,12.8 \mathrm{~Hz}, 1 \mathrm{H}), 2.01(\mathrm{br} \mathrm{d}, J=13.6$ $\mathrm{Hz}, 1 \mathrm{H}), 1.59-1.22(\mathrm{~m}, 5 \mathrm{H}) .{ }^{13} \mathrm{C}\left\{{ }^{1} \mathrm{H}\right\} \mathrm{NMR}: \delta$ 175.6, 171.0, 170.4, 137.9, 133.7, 126.1, 120.9, 119.4, 
119.1, 109.3, 107.3, 59.2, 53.3, 52.1, 42.3, 42.2, 37.4, 33.8, 29.6, 24.6, 23.7, 23.1, 22.2. IR (neat, $\mathrm{cm}^{-1}$ ): 2950, 1732, 1469, 1433, 1260, 1048, 911, 737. Anal. calcd (found) for $\mathrm{C}_{23} \mathrm{H}_{27} \mathrm{NO}_{6}$ : C, $66.81(67.06)$; $\mathrm{H}$, $6.58(6.68)$.

3-Carbomethoxy-9-methyl-2,3,4,9-tetrahydro-1H-carbazol (Table 1, entry 10). ${ }^{7}$ Pale yellow oil, 76\%. TLC (hexanes-EtOAc $=2: 1): R_{f}=0.61 .{ }^{1} \mathrm{H}$ NMR: $\delta 7.48(\mathrm{~d}, J=8.0 \mathrm{~Hz}, 1 \mathrm{H}), 7.25(\mathrm{~d}$, $J=8.4 \mathrm{~Hz}, 1 \mathrm{H}), 7.20(\mathrm{dt}, J=0.8,7.6 \mathrm{~Hz}, 1 \mathrm{H}), 7.11(\mathrm{dt}, J=0.8,7.8 \mathrm{~Hz}, 1 \mathrm{H}), 3.75(\mathrm{~s}, 3 \mathrm{H}), 3.63(\mathrm{~s}, 3 \mathrm{H})$, $3.10(\mathrm{dd}, J=5.4,15.0 \mathrm{~Hz}, 1 \mathrm{H}), 2.96-2.70(\mathrm{~m}, 4 \mathrm{H}), 2.38-2.33(\mathrm{~m}, 1 \mathrm{H}), 2.47(\mathrm{dtd}, J=6.0,10.4,13.2 \mathrm{~Hz}, 1$ H). ${ }^{13} \mathrm{C}\left\{{ }^{1} \mathrm{H}\right\}$ NMR: $\delta 176.3,137.3,134.9,127.1,121.1,119.1,118.0,108.9,107.7,82.1,40.4,29.4$, 26.0, 24.4, 21.5. IR (neat, $\mathrm{cm}^{-1}$ ): 2934, 1731, 1470, 1434, 1416, 1379, 1281, 1166, 1080, 1014, 739.

cis-3-Carbomethoxy-4-ethyl-1,1-dimethyl-2,3,4,9-tetrahydro-1H-carbazole (cis-S7, Table 1, entry 11). $\mathrm{W}_{h}$ ite solid, 92\%. $\mathrm{mp} 64-67^{\circ} \mathrm{C} . \quad \mathrm{TLC}$ (hexanes-EtOAc $=2: 1$ ): $\mathrm{Rf}=0.68 . \quad 1 \mathrm{H}$ NMR (Figure S3): $\delta 7.96(\mathrm{~s}, 1 \mathrm{H}), 7.57(\mathrm{dd}, J=0.8,8.0 \mathrm{~Hz}, 1 \mathrm{H}), 7.32(\mathrm{dd}, J=0.8,8.4 \mathrm{~Hz}, 1 \mathrm{H}), 7.19-7.10(\mathrm{~m}$, $2 \mathrm{H}), 3.82(\mathrm{~s}, 3 \mathrm{H}), 3.43(\mathrm{td}, J=4.8,8.8 \mathrm{~Hz}, 1 \mathrm{H}), 3.08(\mathrm{ddd}, J=2.6,5.0,13.2 \mathrm{~Hz}, 1 \mathrm{H}), 2.23(\mathrm{t}, J=13.6$ $\mathrm{Hz}, 1 \mathrm{H}), 1.94(\mathrm{dd}, J=2.2,13.8 \mathrm{~Hz}, 1 \mathrm{H}), 1.70-1.55(\mathrm{~m}, 2 \mathrm{H}), 1.45(\mathrm{~s}, 3 \mathrm{H}), 1.30(\mathrm{~s}, 3 \mathrm{H}), 1.06(\mathrm{t}, J=7.6$

$\mathrm{Hz}, 3 \mathrm{H}) .{ }^{13} \mathrm{C}\left\{{ }^{1} \mathrm{H}\right\}$ NMR (Figure S4): $\delta \quad 175.9,141.2,136.3,127.8,121.5,119.5,119.4,112.6,110.9$, 51.9, 42.9, 36.4, 36.3, 32.0, 30.3, 29.7, 26.7, 13.8. IR (neat, $\mathrm{cm}^{-1}$ ): 3406, 2958, 1727, 1713, 1455, 1430, 1140, 1013, 908, 734. HRMS calcd (found) for $\mathrm{C}_{18} \mathrm{H}_{23} \mathrm{NO}_{2}\left(\mathrm{M}^{+}\right)$: 285.1729 (285.1722).

trans-3-Carbomethoxy-4-ethyl-1,1-dimethyl-2,3,4,9-tetrahydro-1H-carbazole （trans-S7,

Table 1, entry 12). Pale yellow viscous oil, 84\%. TLC (hexanes-EtOAc $=2: 1$ ): $R_{f}=0.66 .{ }^{1} \mathrm{H}$ NMR (Figure S5): $\delta 7.96(\mathrm{~s}, 1 \mathrm{H}), 7.62(\mathrm{~d}, J=8.0 \mathrm{~Hz}, 1 \mathrm{H}), 7.32(\mathrm{dd}, J=0.8,8.2 \mathrm{~Hz}, 1 \mathrm{H}), 7.18-7.08(\mathrm{~m}, 2 \mathrm{H})$, 3.80 (s, $3 \mathrm{H}), 3.55$ (ddd, $J=3.0,6.6,9.2 \mathrm{~Hz}, 1 \mathrm{H}), 2.96(\mathrm{ddd}, J=2.4,9.2,12.4 \mathrm{~Hz}, 1 \mathrm{H}), 2.24-2.13$ (m, 1 H), $2.02(\mathrm{t}, J=13.2 \mathrm{~Hz}, 1 \mathrm{H}), 1.97-1.89(\mathrm{~m}, 2 \mathrm{H}), 1.39(\mathrm{~s}, 3 \mathrm{H}), 1.37(\mathrm{~s}, 3 \mathrm{H}), 0.83(\mathrm{t}, J=7.6 \mathrm{~Hz}, 3 \mathrm{H})$. ${ }^{13} \mathrm{C}\left\{{ }^{1} \mathrm{H}\right\}$ NMR (Figure S6): $\delta 177.1,141.8,136.4,127.3,121.3,119.8,119.5,111.0,109.7,52.1,42.7$, 42.1, 36.9, 31.7, 29.4, 29.3, 25.2, 9.3. IR (neat, $\mathrm{cm}^{-1}$ ): 3391, 2961, 1717, 1455, 1455, 1167, $908,740$. HRMS calcd (found) for $\mathrm{C}_{18} \mathrm{H}_{23} \mathrm{NO}_{2}\left(\mathrm{M}^{+}\right)$: 285.1729 (285.1732). 
The stereochemistry of cis-S7 and trans-S7 were established by ${ }^{1} \mathrm{H}$ NOE analysis. In particular, Irradiation of $\mathrm{H}_{4}$ led to the enhancement of $\mathrm{H}_{3}$ in cis-S7 but not in trans-S7. The stereochemistry of cisS7 was confirmed via X-ray crystallographic analysis (Tables S2-S5, Figure S7).
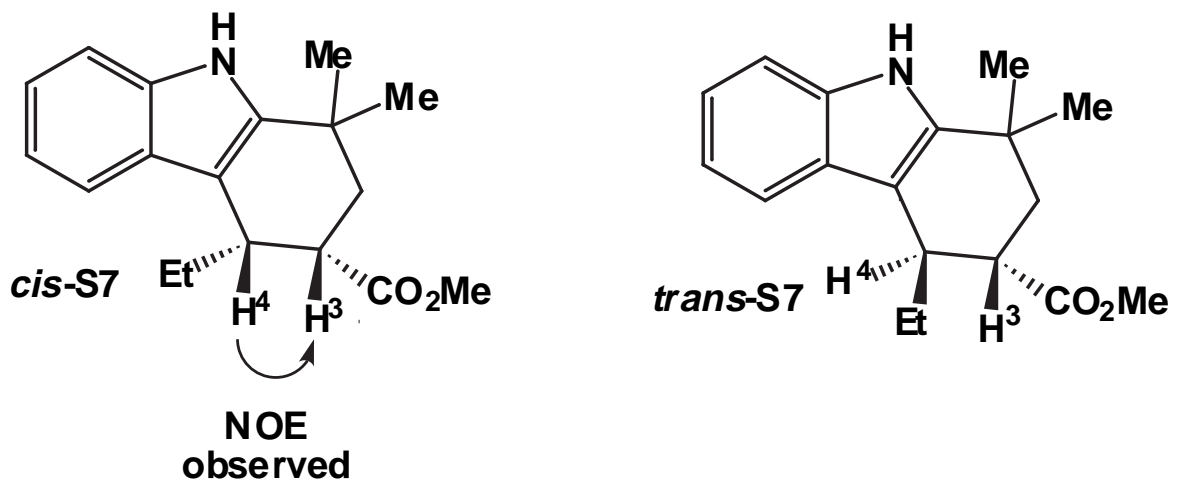

Methyl (5-methyl-5,6,7,8,9,10-hexahydro-cyclohepta[b]indol-10-yl)-acetate (Table 1, entry 13). Pale yellow oil, 74\%. TLC (hexanes-EtOAc $=2: 1): R_{f}=0.70 .{ }^{1} \mathrm{H}$ NMR: $\delta \quad 7.60(\mathrm{~d}, J=7.6$ $\mathrm{Hz}, 1 \mathrm{H}), 7.25(\mathrm{~d}, J=8.0 \mathrm{~Hz}, 1 \mathrm{H}), 7.19(\mathrm{dt}, J=1.0,7.6 \mathrm{~Hz}, 1 \mathrm{H}), 7.12(\mathrm{dt}, J=0.8,7.6 \mathrm{~Hz}, 1 \mathrm{H}), 3.93-3.88$ (m, $1 \mathrm{H}), 3.67$ (s, $3 \mathrm{H}), 3.65$ (s, $3 \mathrm{H}), 3.03$ (ddd, $J=2.4,6.2,16.0 \mathrm{~Hz}, 1 \mathrm{H}), 2.81$ (ddd, $J=2.4,12.0,15.6$ $\mathrm{Hz}, 1 \mathrm{H}), 2.68(\mathrm{~d}, J=8.0 \mathrm{~Hz}, 2 \mathrm{H}), 2.13-1.59(\mathrm{~m}, 6 \mathrm{H}) .{ }^{13} \mathrm{C}\left\{{ }^{1} \mathrm{H}\right\} \mathrm{NMR}: \delta \quad 173.7,138.7,136.1,127.4$, 120.8, 119.1, 118.0, 115.4, 109.1, 51.8, 38.8, 31.9, 31.8, 29.7, 27.7, 25.9, 25.6. IR (neat, $\left.\mathrm{cm}^{-1}\right): 2922,2852$, $1730,1470,1434,1382,1360,1285,1165,1015,739$. Anal. calcd (found) for $\mathrm{C}_{17} \mathrm{H}_{21} \mathrm{NO}_{2}$ : $\mathrm{C}, 75.25$ (75.24); H, 7.80 (7.75).

2-Carbomethoxy-9-methyl-2,3,4,9-tetrahydro-1H-carbazole (Table 1, entry 14). ${ }^{7}$ Pale yellow solid, 58\%. TLC (hexanes-EtOAc $=2: 1): R_{f}=0.61 .{ }^{1} \mathrm{H}$ NMR: $\delta 7.50(\mathrm{~d}, J=7.6 \mathrm{~Hz}, 1 \mathrm{H}), 7.29$ $(\mathrm{d}, J=8.4 \mathrm{~Hz}, 1 \mathrm{H}), 7.15(\mathrm{dt}, J=0.8,7.8 \mathrm{~Hz}, 1 \mathrm{H}), 7.13(\mathrm{dt}, J=1.0,7.8 \mathrm{~Hz}, 1 \mathrm{H}), 3.81(\mathrm{~s}, 3 \mathrm{H}), 3.66(\mathrm{~s}, 3$ $\mathrm{H}), 3.04-2.75(\mathrm{~m}, 5 \mathrm{H}), 2.40-2.34(\mathrm{~m}, 1 \mathrm{H}), 2.47(\mathrm{dtd}, J=5.6,10.8,13.2 \mathrm{~Hz}, 1 \mathrm{H}) .{ }^{13} \mathrm{C}\left\{{ }^{1} \mathrm{H}\right\} \mathrm{NMR}: \quad \delta$ 175.9, 137.3, 134.0, 127.0, 121.1, 119.1, 118.1, 108.9, 108.8, 52.2, 40.6, 29.4, 26.6, 24.9, 20.7. IR (neat, 
$\mathrm{cm}^{-1}$ ): 2948, 2846, 1742, 1732, 1470, 1380, 1168, 1380, 1168, 1011, 739. Anal. calcd (found) for $\mathrm{C}_{15} \mathrm{H}_{17} \mathrm{NO}_{2}: \mathrm{C}, 74.05$ (73.96); H, 7.04 (7.17).

Methyl (2,3,4,9-tetrahydro-1H-carbazol-1-yl)-acetate (Table 1, entry 15). Pale yellow oil, 85\%. TLC (hexanes-EtOAc $=5: 1): R_{f}=0.64 .{ }^{1} \mathrm{H}$ NMR: $\delta 8.74(\mathrm{~s}, 1 \mathrm{H}), 7.51(\mathrm{dd}, J=0.8,8.4 \mathrm{~Hz}, 1 \mathrm{H})$, $7.33(\mathrm{td}, J=0.8,8.2 \mathrm{~Hz}, 1 \mathrm{H}), 7.17(\mathrm{dt}, J=1.0,7.6 \mathrm{~Hz}, 1 \mathrm{H}), 7.11(\mathrm{dt}, J=1.0,7.6 \mathrm{~Hz}, 1 \mathrm{H}), 3.80(\mathrm{~s}, 3 \mathrm{H})$, 3.43-3.36 (m, $1 \mathrm{H}), 2.76-2.63(\mathrm{~m}, 4 \mathrm{H}), 2.13-2.06(\mathrm{~m}, 1 \mathrm{H}), 2.00-1.82(\mathrm{~m}, 2 \mathrm{H}), 1.72-1.65(\mathrm{~m}, 1 \mathrm{H})$. ${ }^{13} \mathrm{C}\left\{{ }^{1} \mathrm{H}\right\}$ NMR: $\delta$ 175.3, 136.5, 135.9, 127.5, 121.6, 119.3, 118.3, 111.1, 110.5, 52.3, 40.6, 30.9, 30.2, 21.7, 21.3. IR (neat, $\mathrm{cm}^{-1}$ ): 3406, 2930, 2846, 1727, 1466, 1363, 1329, 1009, 741. Anal. calcd (found) for $\mathrm{C}_{15} \mathrm{H}_{17} \mathrm{NO}_{2}: \mathrm{C}, 74.05$ (73.90); H, 7.04 (7.07).

\section{THF as solvent}

S6. THF (4 mL), methanol (180 $\mu \mathrm{L}, 5.0 \mathrm{mmol})$, and a solution of $\mathbf{S 2}(99 \mathrm{mg}, 0.50 \mathrm{mmol})$ in THF $(1.0 \mathrm{~mL})$ were added sequentially to a flask containing $2(6.5 \mathrm{mg}, 0.025 \mathrm{mmol})$ and $\mathrm{CuCl}_{2}(200 \mathrm{mg}, 1.5$ mmol) under CO (1 atm). The resulting suspension was stirred for $30 \mathrm{~min}$ and concentrated under vacuum. Column chromatography of the residue $\left(\mathrm{SiO}_{2} ;\right.$ hexanes-EtOAc $\left.=5: 1\right)$ gave $\mathbf{S 6}(106 \mathrm{mg}, 83 \%)$ as a pale yellow oil. Employment of fewer than ten equivalents of methanol led to diminished yields of S6.

The remaining tricyclic indole derivatives depicted in Scheme 2 were synthesized from S2 employing a procedure similar to that used to synthesize S6.

Ethyl (2,3,4,9-tetrahydro-1H-carbazol-4-yl)-acetate. Pale yellow oil, 73\%. TLC (hexanesEtOAc $=2: 1): R_{f}=0.58 .{ }^{1} \mathrm{H}$ NMR: $\delta 7.78(\mathrm{~s}, 1 \mathrm{H}), 7.52(\mathrm{~d}, J=8.4 \mathrm{~Hz}, 1 \mathrm{H}), 7.26-7.23(\mathrm{~m}, 1 \mathrm{H}), 7.14-$ $7.06(\mathrm{~m}, 1 \mathrm{H}), 4.20(\mathrm{q}, J=7.2 \mathrm{~Hz}, 2 \mathrm{H}), 3.58-3.52(\mathrm{~m}, 1 \mathrm{H}), 2.99(\mathrm{dd}, J=4.0,15.2 \mathrm{~Hz}, 1 \mathrm{H}), 2.69-2.66$ (m, $2 \mathrm{H}), 2.41(\mathrm{dd}, J=10.4,14.8 \mathrm{~Hz}, 1 \mathrm{H}), 2.00-1.74(\mathrm{~m}, 4 \mathrm{H}), 1.29(\mathrm{t}, J=6.8 \mathrm{~Hz}, 3 \mathrm{H}) .{ }^{13} \mathrm{C}\left\{{ }^{1} \mathrm{H}\right\} \mathrm{NMR}: \delta$ 173.6, 136.0, 134.9, 127.1, 121.3, 119.5, 118.4, 112.4, 110.9, 60.6, 40.2, 29.3, 28.7, 23.5, 19.7, 14.6. IR $\left(\right.$ neat, $\left.\mathrm{cm}^{-1}\right): 3399,2932,1729,1713,1463,1370,1326,1301,1281,1177,1071,1030,741$. Anal. calcd 
(found) for $\mathrm{C}_{16} \mathrm{H}_{19} \mathrm{NO}_{2}$ : C, 74.68 (74.40); $\mathrm{H}, 7.44$ (7.65).

$\boldsymbol{n}$-Octyl (2,3,4,9-tetrahydro-1H-carbazol-4-yl)-acetate. Pale yellow oil, 82\%. TLC (hexanesEtOAc $=2: 1): R_{f}=0.75 .{ }^{1} \mathrm{H}$ NMR: $\delta 7.78(\mathrm{~s}, 1 \mathrm{H}), 7.53(\mathrm{~d}, J=8.4 \mathrm{~Hz}, 1 \mathrm{H}), 7.26-7.24(\mathrm{~m}, 1 \mathrm{H}), 7.14-$ $7.06(\mathrm{~m}, 1 \mathrm{H}), 4.14(\mathrm{t}, J=6.2 \mathrm{~Hz}, 2 \mathrm{H}), 3.58-3.53(\mathrm{~m}, 1 \mathrm{H}), 2.99(\mathrm{dd}, J=4.0,15.2 \mathrm{~Hz}, 1 \mathrm{H}), 2.69-2.67(\mathrm{~m}$, 2 H), 2.42 (dd, $J=10.4,14.8 \mathrm{~Hz}, 1 \mathrm{H}), 1.98-1.73$ (m, 4 H), 1.68-1.62 (m, $2 \mathrm{H}), 1.39-1.26$ (m, $10 \mathrm{H}), 0.91$ $(\mathrm{t}, J=6.8 \mathrm{~Hz}, 3 \mathrm{H}) .{ }^{13} \mathrm{C}\left\{{ }^{1} \mathrm{H}\right\}$ NMR: $\delta$ 173.7, 136.1, 134.9, 127.1, 121.3, 119.5, 118.4, 112.4, 110.9, 64.9, 40.3, 32.1, 29.5, 29.4, 28.9, 28.8, 26.2, 23.5, 23.0, 19.7, 14.4. IR (neat, $\left.\mathrm{cm}^{-1}\right)$ : 3402, 2925, 2855, 1713, 1454, 739. Anal. calcd (found) for $\mathrm{C}_{22} \mathrm{H}_{31} \mathrm{NO}_{2}$ : C, 77.38 (77.13); $\mathrm{H}, 9.15$ (9.23).

Isopropyl (2,3,4,9-tetrahydro-1H-carbazol-4-yl)-acetate. Pale yellow oil, $75 \%$. TLC (hexanes-EtOAc $=2: 1): \quad R_{f}=0.61 .{ }^{1} \mathrm{H}$ NMR (Figure S8): $\delta 7.79(\mathrm{~s}, 1 \mathrm{H}), 7.54(\mathrm{~d}, J=7.6 \mathrm{~Hz}, 1 \mathrm{H}$ ), 7.26-7.23 (m, $1 \mathrm{H}), 7.15-7.06(\mathrm{~m}, 1 \mathrm{H}), 5.10$ (septet, $J=7.6 \mathrm{~Hz}, 1 \mathrm{H}), 3.58-3.53(\mathrm{~m}, 1 \mathrm{H}), 2.99$ (dd, $J=$ 4.0, $15.2 \mathrm{~Hz}, 1 \mathrm{H}), 2.70-2.62(\mathrm{~m}, 2 \mathrm{H}), 2.39$ (dd, $J=10.4,14.8 \mathrm{~Hz}, 1 \mathrm{H}), 2.00-1.76(\mathrm{~m}, 4 \mathrm{H}), 1.29$ (d, $J=$ $6.4 \mathrm{~Hz}, 3 \mathrm{H}$ ), $1.26(\mathrm{~d}, J=6.4 \mathrm{~Hz}, 3 \mathrm{H}) .{ }^{13} \mathrm{C}\left\{{ }^{1} \mathrm{H}\right\}$ NMR (Figure S9): $\delta$ 173.2, 136.0, 134.9, 127.1, 121.3, 119.4, 118.5, 112.4, 110.8, 67.9, 40.6, 29.4, 28.7, 23.5, 22.3, 22.1, 19.8. IR (neat, $\left.\mathrm{cm}^{-1}\right)$ : 3402, 2978, 2934, 1729, 1453, 1372, 1107, 741. HRMS calcd (found) for $\mathrm{C}_{17} \mathrm{H}_{21} \mathrm{NO}_{2}\left(\mathrm{M}^{+}\right)$: 271.1572 (271.1568).

Cyclohexyl (2,3,4,9-tetrahydro-1H-carbazol-4-yl)-acetate. Pale yellow oil, 73\%. TLC (hexanes-EtOAc $=2: 1): R_{f}=0.71 .{ }^{1} \mathrm{H}$ NMR (Figure S10): $\delta 7.78(\mathrm{~s}, 1 \mathrm{H}), 7.56(\mathrm{~d}, J=7.6 \mathrm{~Hz}, 1 \mathrm{H})$, 7.29-7.27 (m, $1 \mathrm{H}), 7.15-7.07$ (m, $2 \mathrm{H}), 4.86(\mathrm{tt}, J=3.6,8.8 \mathrm{~Hz}, 1 \mathrm{H}), 3.59-3.52(\mathrm{~m}, 1 \mathrm{H}), 2.99$ (dd, $J=4.0$, $15.2 \mathrm{~Hz}, 1 \mathrm{H}), 2.72-2.69$ (m, $2 \mathrm{H}), 2.41(\mathrm{dd}, J=10.4,14.8 \mathrm{~Hz}, 1 \mathrm{H}), 1.99-1.69(\mathrm{~m}, 8 \mathrm{H}), 1.58-1.27$ (m, 6 H). ${ }^{13} \mathrm{C}\left\{{ }^{1} \mathrm{H}\right\}$ NMR (Figure S11): $\delta$ 173.1, 136.1, 134.9, 127.1, 121.3, 119.5, 118.5, 112.5, 110.9, 72.8, 40.6, 32.0, 31.9, 29.5, 28.7, 25.7, 24.0, 23.5, 19.8. IR (neat, $\mathrm{cm}^{-1}$ ): 3403, 2935, 1725, 1453, 1281, 1182, 1014, 908, 741. HRMS calcd (found) for $\mathrm{C}_{20} \mathrm{H}_{25} \mathrm{NO}_{2}\left(\mathrm{M}^{+}\right)$: 311.1885 (311.1887). 
Figure S1. ${ }^{1} \mathrm{H}$ NMR spectrum of methyl (6-fluoro-2,3,4,9-tetrahydro-1H-carbazol-4-yl)-acetate (Table 1, entry 3 ).

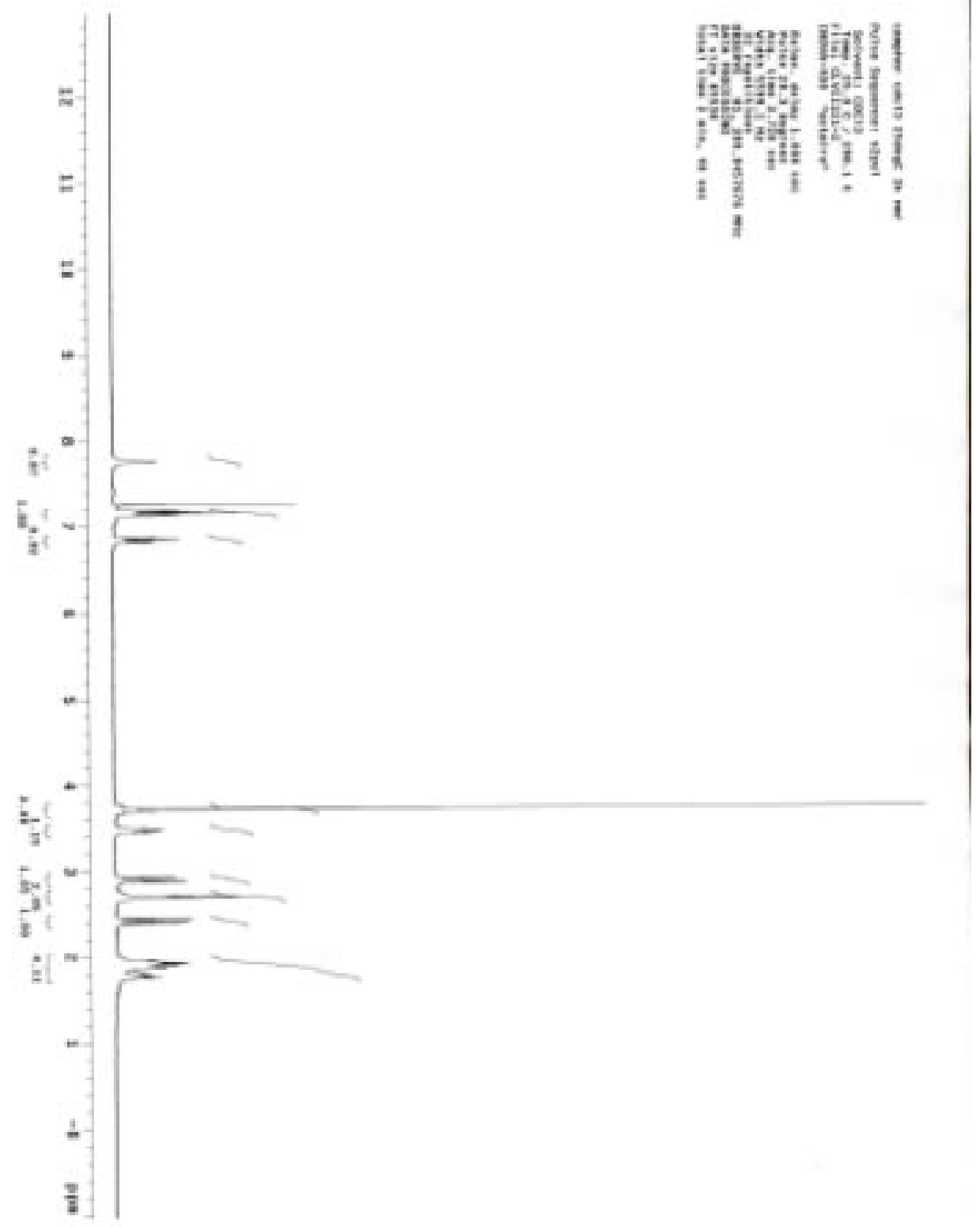


Figure S2. ${ }^{13} \mathrm{C}\left\{{ }^{1} \mathrm{H}\right\}$ NMR spectrum of methyl (6-fluoro-2,3,4,9-tetrahydro- $1 H$-carbazol-4-yl)-acetate (Table 1, entry 3).
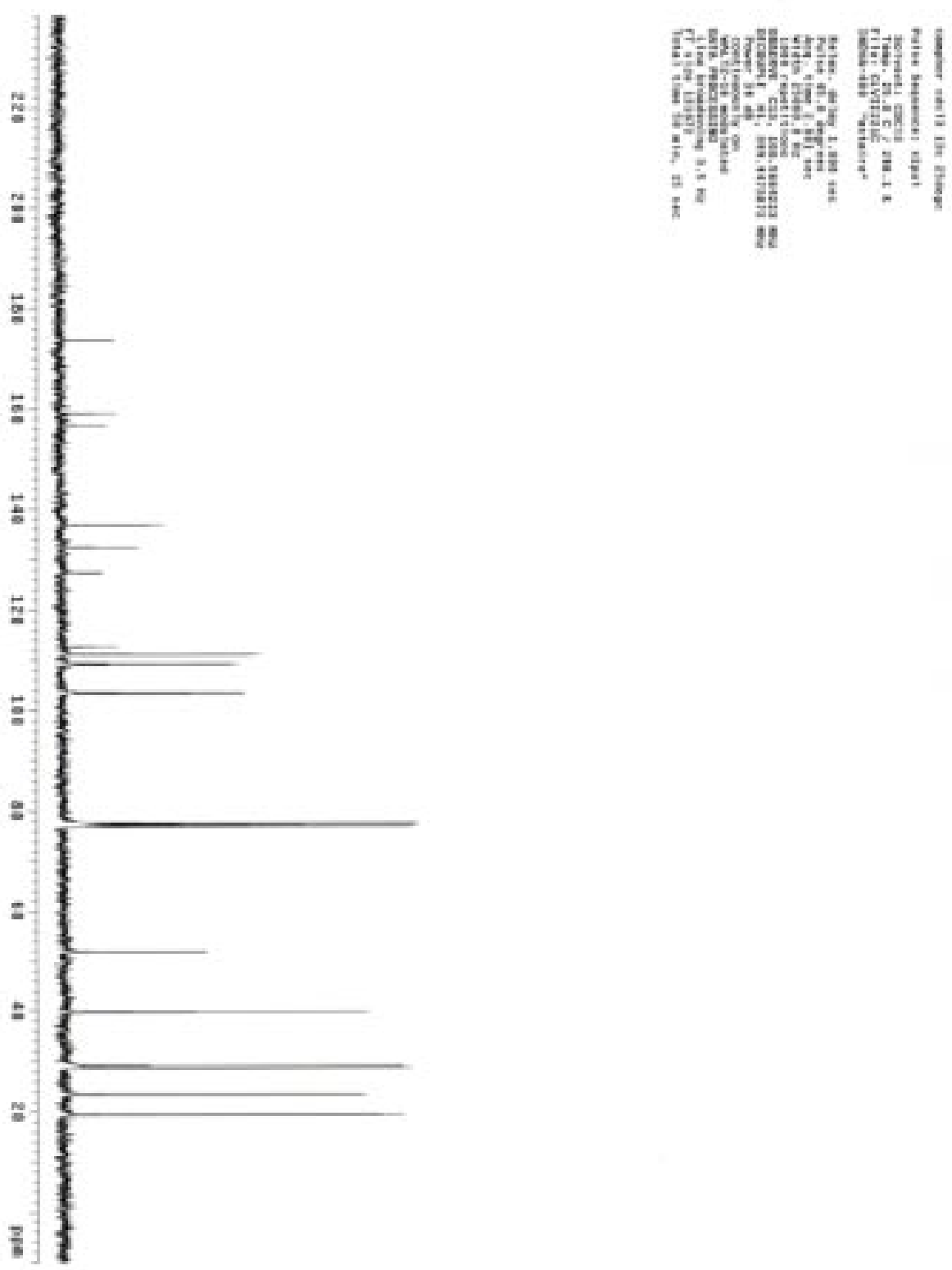
Figure S3. ${ }^{1}$ H NMR spectrum of cis-S7 (Table 1, entry 11).

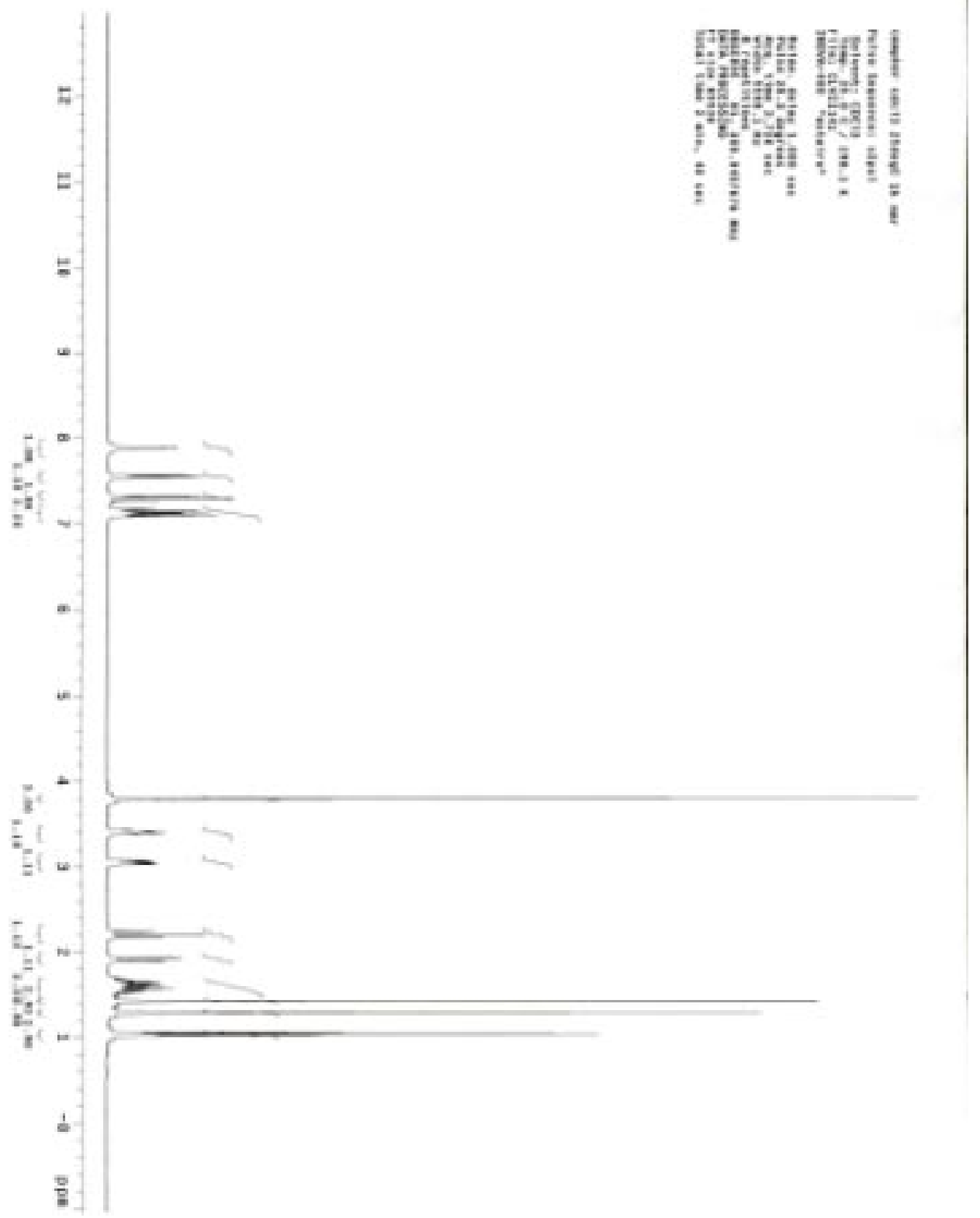


Figure S4. ${ }^{13} \mathrm{C}\left\{{ }^{1} \mathrm{H}\right\}$ NMR spectrum of $c$ is-S7 (Table 1, entry 11).

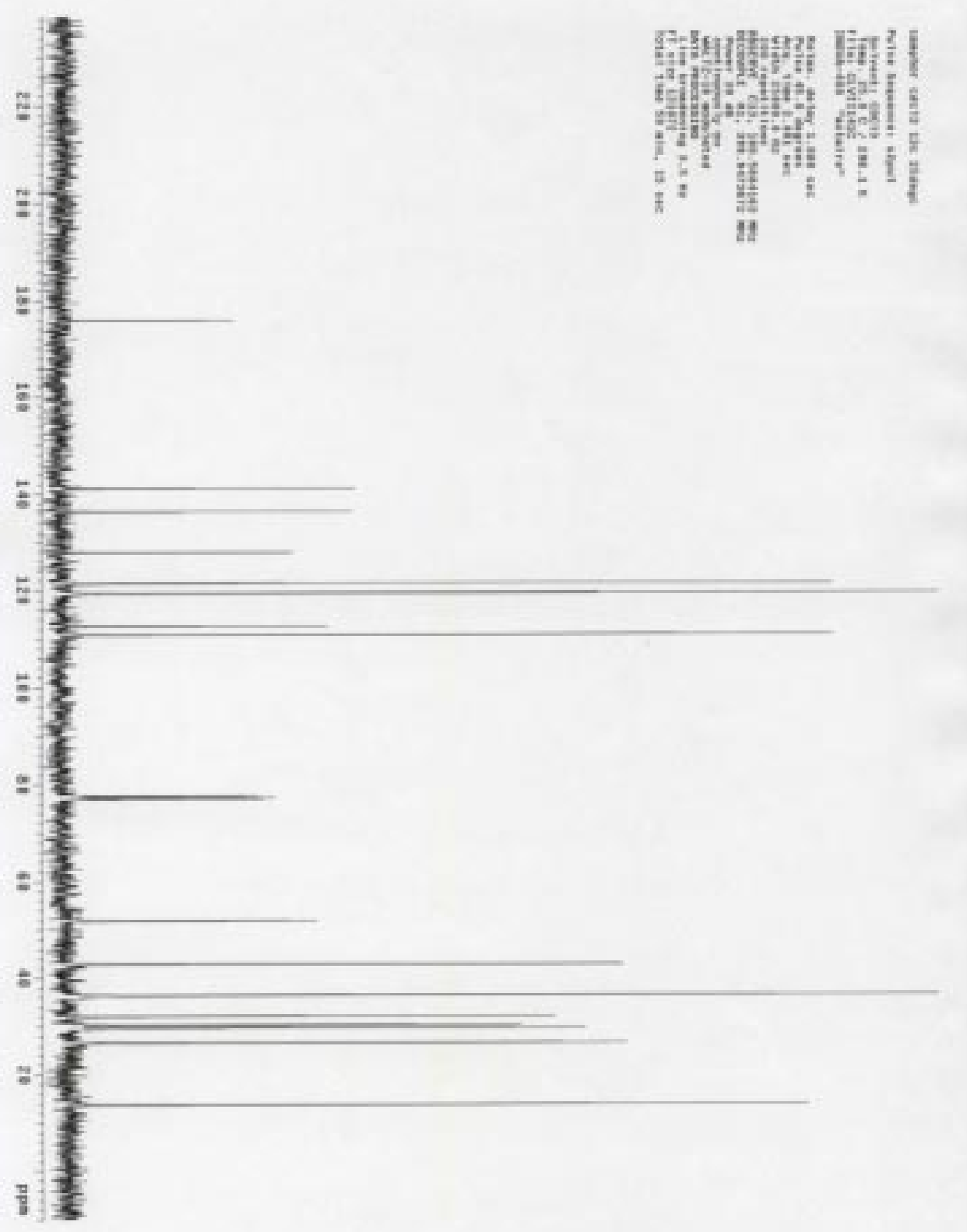


Figure S5. ${ }^{1} \mathrm{H}$ NMR spectrum of trans-S7 (Table 1, entry 12).

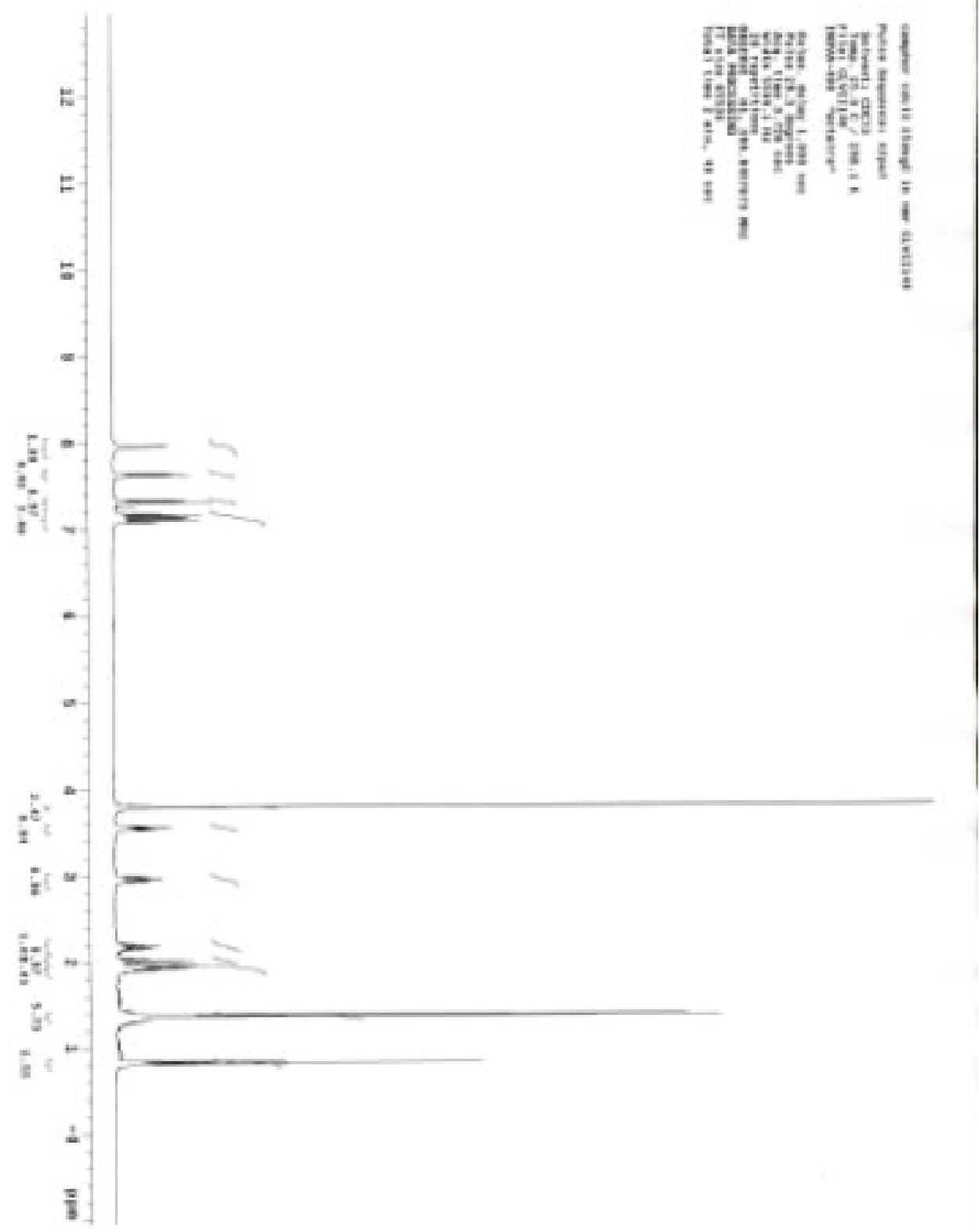


Figure S6. ${ }^{13} \mathrm{C}\left\{{ }^{1} \mathrm{H}\right\}$ NMR spectrum of trans-S7 (Table 1, entry 12).

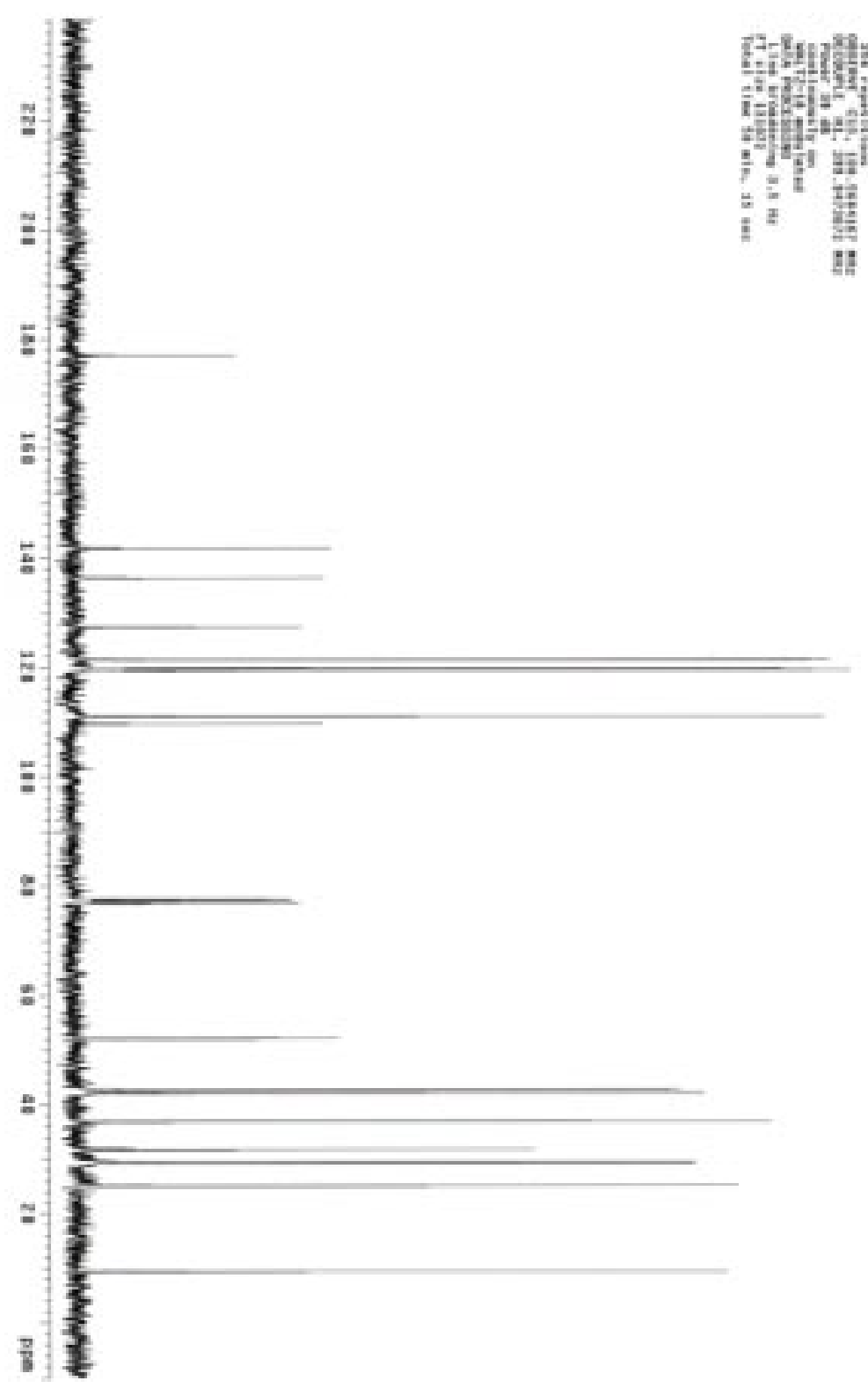


Figure S7. X-ray crystal structure of $c i s-S 7$.

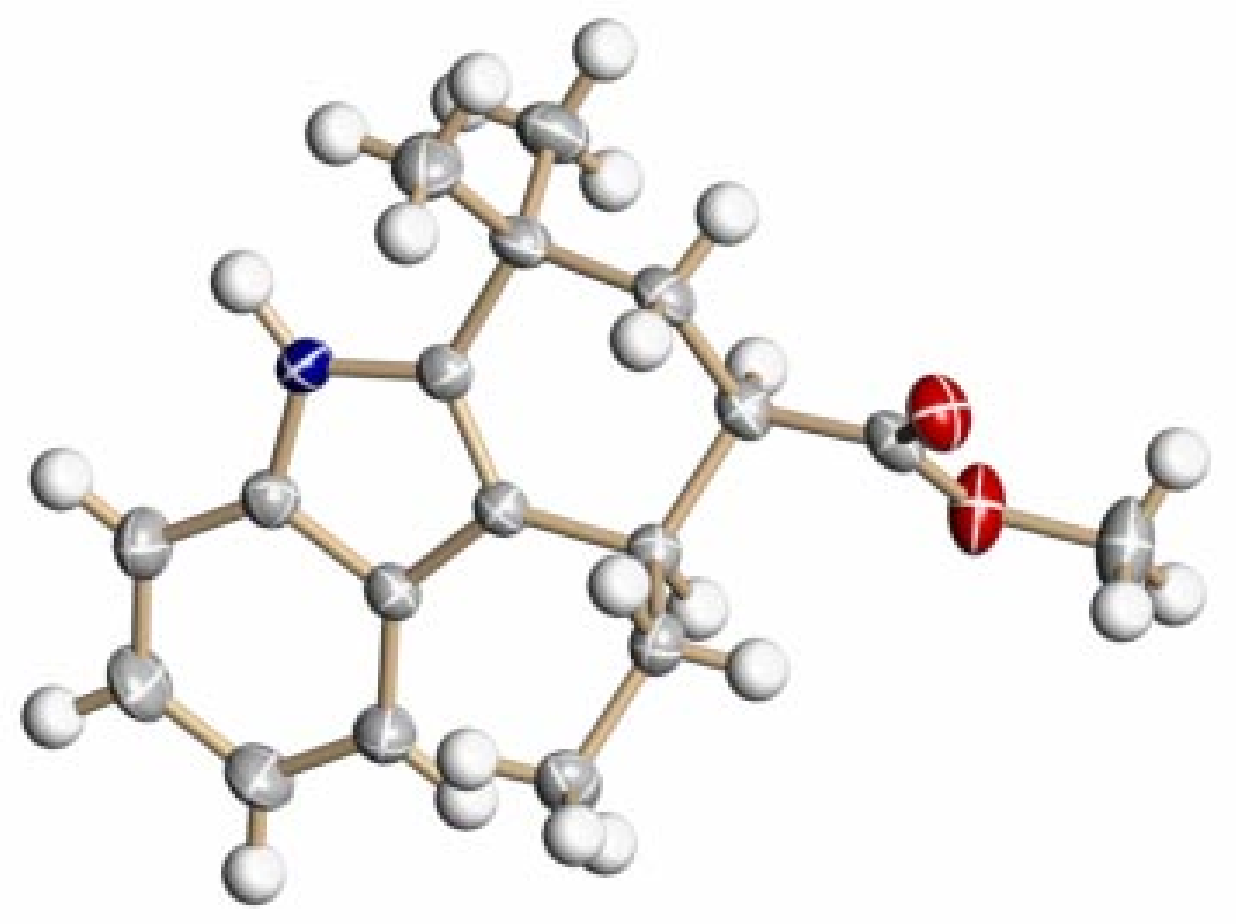


Figure S8. ${ }^{1}$ H NMR spectrum of isopropyl (2,3,4,9-tetrahydro-1H-carbazol-4-yl)-acetate (Scheme 2).

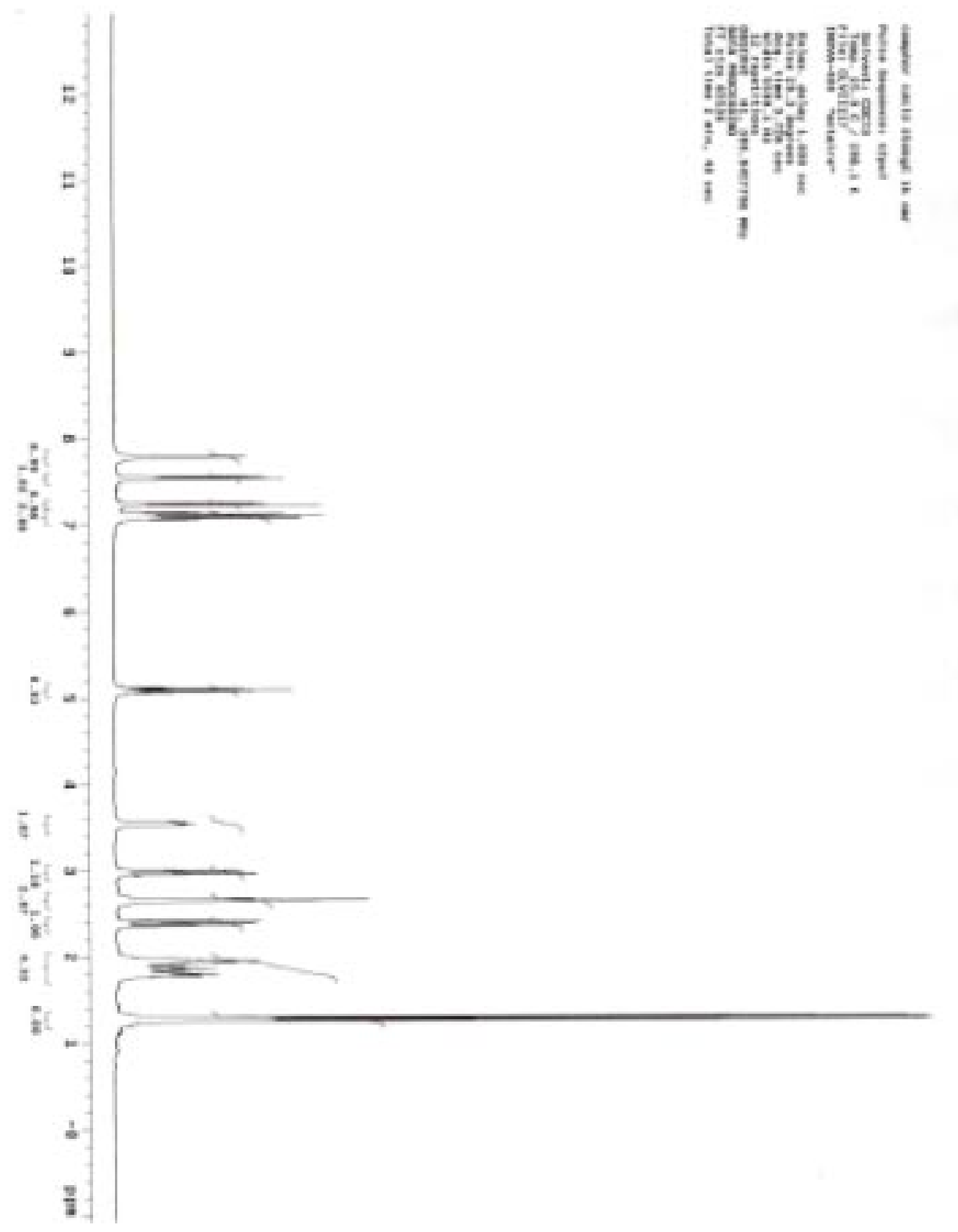


Figure S9. ${ }^{13} \mathrm{C}\left\{{ }^{1} \mathrm{H}\right\}$ NMR spectrum of isopropyl (2,3,4,9-tetrahydro-1H-carbazol-4-yl)-acetate (Scheme 2).

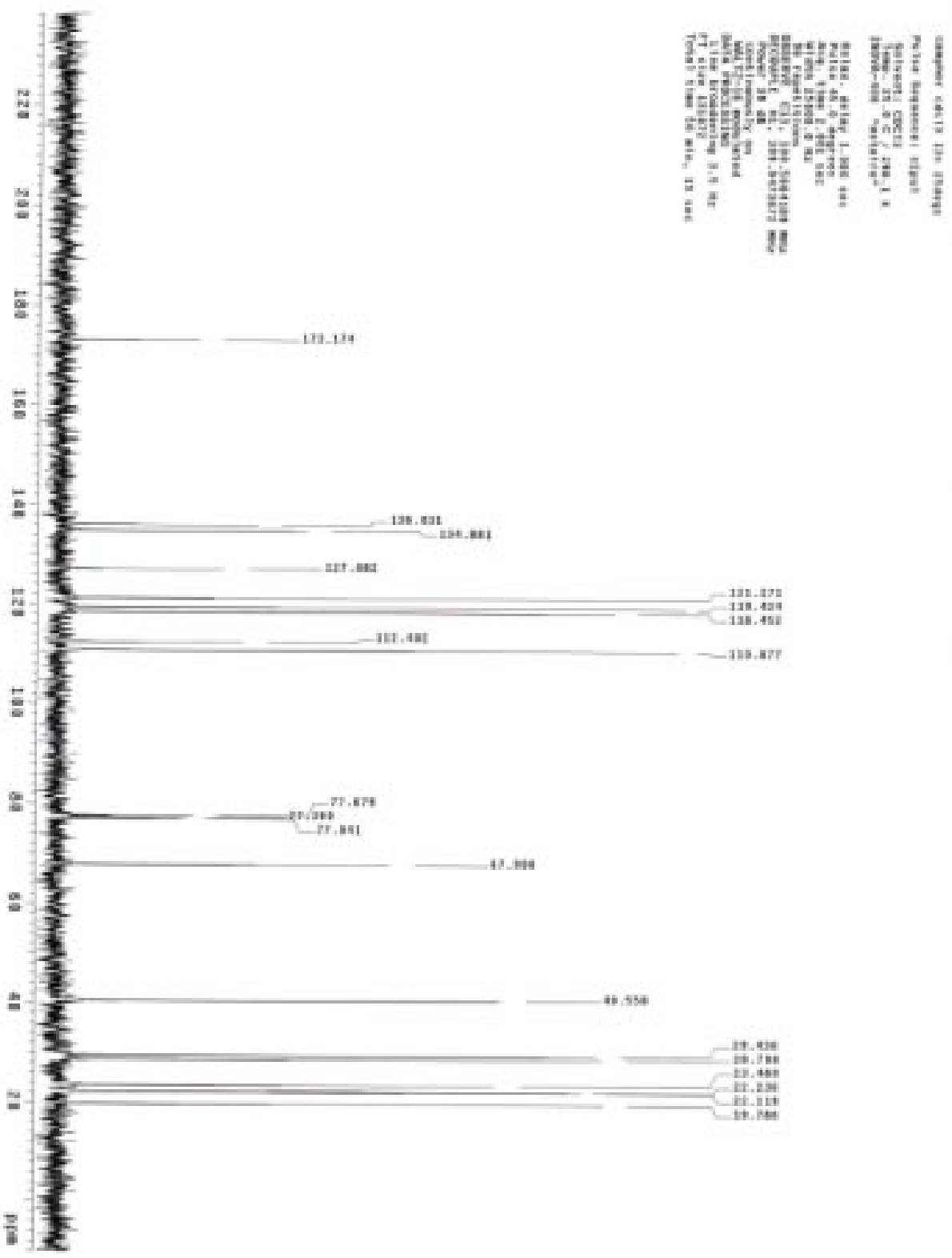


Figure S10. ${ }^{1}$ H NMR spectrum of cyclohexyl (2,3,4,9-tetrahydro-1H-carbazol-4-yl)-acetate (Scheme 2).
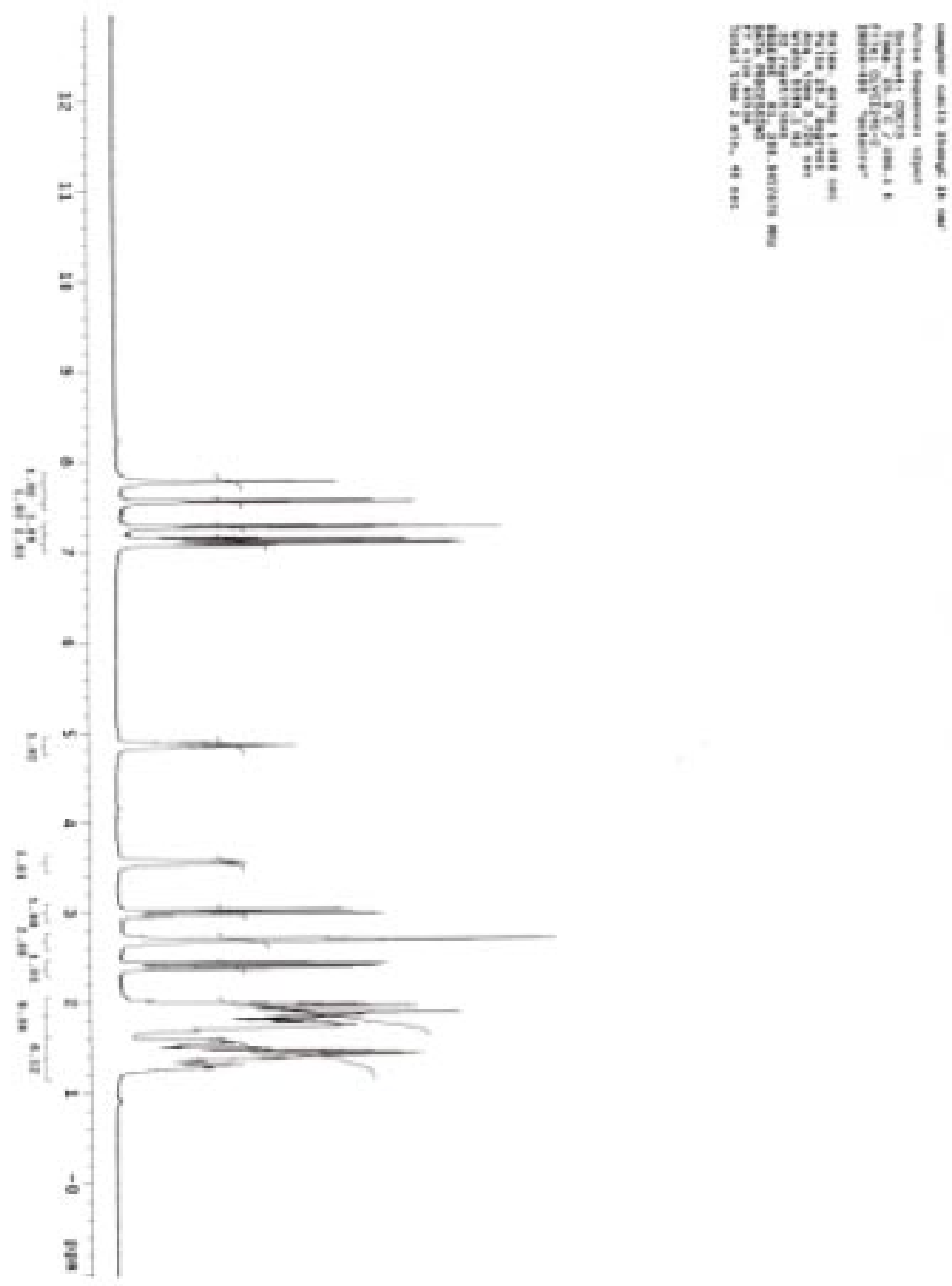
Figure S11. ${ }^{13} \mathrm{C}\left\{{ }^{1} \mathrm{H}\right\}$ NMR spectrum of cyclohexyl (2,3,4,9-tetrahydro- $1 H$-carbazol-4-yl)-acetate (Scheme 2).

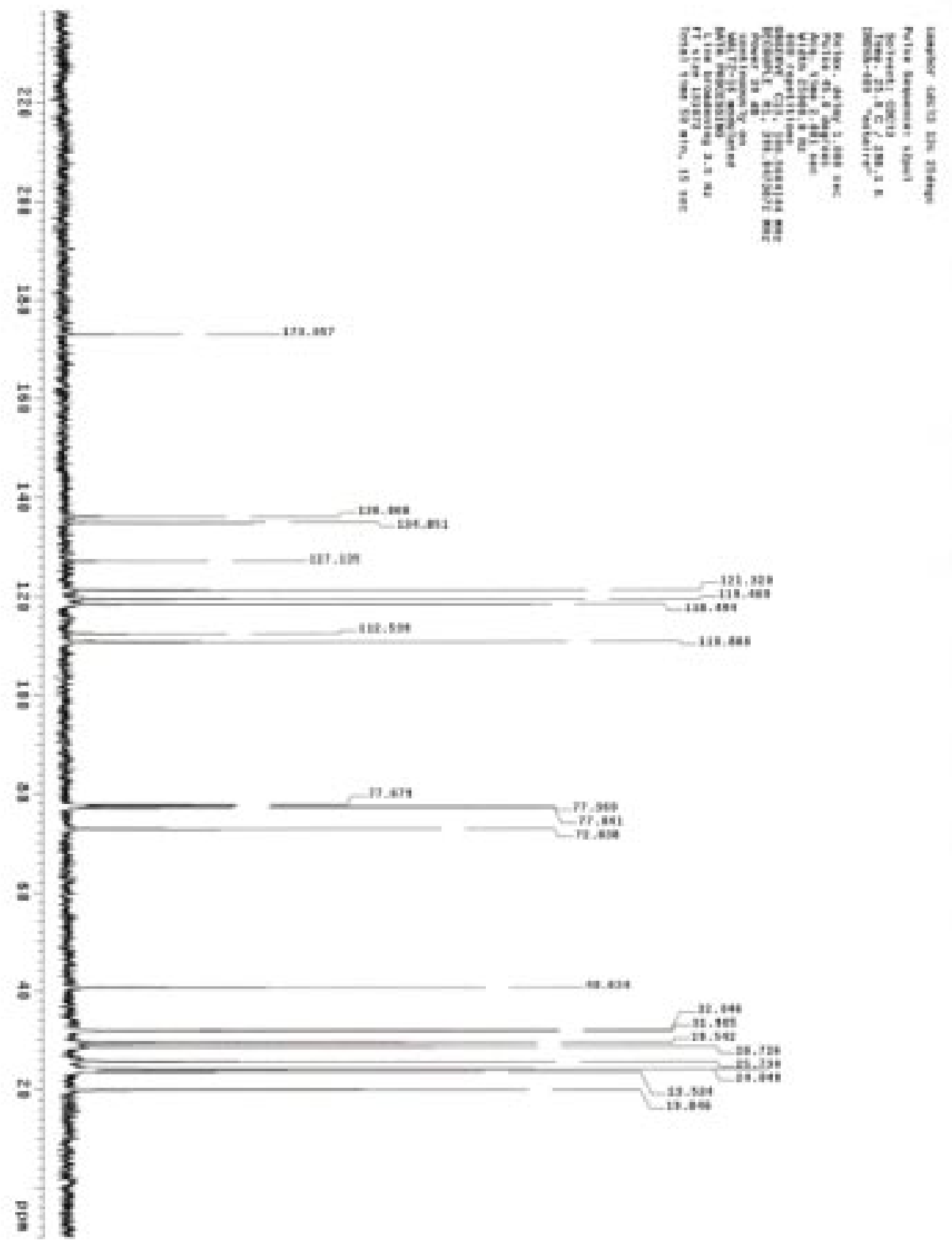


Table S2. X-ray crystal data and collection and refinement of parameters for cis-S7.

\begin{tabular}{|c|c|}
\hline $\begin{array}{l}\text { empiric al formula } \\
\text { fw }\end{array}$ & $\begin{array}{l}\mathrm{C}_{18} \mathrm{H}_{23} \mathrm{NO}_{2} \\
285.38\end{array}$ \\
\hline crystal size (mm) & $0.30 \times 0.30 \times 0.20$ \\
\hline crystal sy stem & Orthorhombic \\
\hline space group & P bca \\
\hline$a(\AA)$ & $9.4635(4)$ \\
\hline$b(\AA)$ & $16.8018(7)$ \\
\hline$c(\AA)$ & $20.2194(9)$ \\
\hline$V\left(\AA^{3}\right)$ & $3214.96(24)$ \\
\hline$z$ & 8 \\
\hline scan mode & w \\
\hline $2 \theta$ limits (deg) & $5.00<2 q<50.00$ \\
\hline$D_{c}\left(\mathrm{mg}^{*} \mathrm{~cm}^{-3}\right)$ & 1.179 \\
\hline unique reflections & 2842 \\
\hline data with Inet $>25 \sigma(I)$ & 2151 \\
\hline$F(000)$ & 1232.69 \\
\hline$R_{f}$ & 0.044 \\
\hline$R_{w}$ & 0.049 \\
\hline GoF & 1.9942 \\
\hline No. of pa rameters & 191 \\
\hline $\max \Delta / \sigma$ & 0.000 \\
\hline largest resid density $\left(\mathrm{e} / \AA^{3}\right)$ & 0.230 \\
\hline
\end{tabular}


Table S3. Atomic coordinates for $c i s-$ S7.

$\begin{array}{lllll} & \mathrm{x} & \mathrm{y} & \mathrm{z} & \mathrm{Biso} \\ \mathrm{C} 1 & 0.02874(17) & 0.79392(10) & 0.11622(9) & 1.68(7) \\ \mathrm{C} 2 & -0.05462(18) & 0.72554(10) & 0.09752(9) & 1.81(7) \\ \mathrm{C} 3 & -0.19807(19) & 0.71188(11) & 0.08345(10) & 2.31(8) \\ \mathrm{C} 4 & -0.24171(20) & 0.63628(12) & 0.06763(11) & 2.90(9) \\ \mathrm{C} 5 & -0.14632(22) & 0.57284(12) & 0.06497(11) & 3.22(10) \\ \mathrm{C} 6 & -0.00548(22) & 0.58352(11) & 0.07885(10) & 2.79(9) \\ \mathrm{C} 7 & 0.03926(19) & 0.66002(10) & 0.09542(9) & 2.01(7) \\ \mathrm{N} 8 & 0.17156(15) & 0.68707(9) & 0.11287(8) & 2.02(6) \\ \mathrm{C} 9 & 0.16402(18) & 0.76754(10) & 0.12567(9) & 1.77(8) \\ \mathrm{C} 10 & 0.28773(18) & 0.81435(11) & 0.15173(10) & 2.09(7) \\ \mathrm{C} 11 & 0.24555(19) & 0.90297(10) & 0.15059(10) & 2.19(8) \\ \mathrm{C} 12 & 0.09426(18) & 0.91798(10) & 0.17392(9) & 1.89(7) \\ \mathrm{C} 13 & -0.01612(17) & 0.87909(10) & 0.12696(9) & 1.73(7) \\ \mathrm{C} 14 & 0.42010(20) & 0.80247(12) & 0.10921(11) & 3.05(10) \\ \mathrm{C} 15 & 0.31926(22) & 0.78672(12) & 0.22280(11) & 3.09(9) \\ \mathrm{C} 16 & 0.06414(19) & 1.00519(11) & 0.18294(9) & 2.00(7) \\ \mathrm{O} 17 & 0.13272(13) & 1.05963(7) & 0.16040(7) & 2.70(6) \\ \mathrm{O} 18 & -0.05199(14) & 1.01646(8) & 0.21922(7) & 3.09(6) \\ \mathrm{C} 19 & -0.09264(24) & 1.09858(12) & 0.23161(12) & 3.71(10) \\ \mathrm{C} 20 & -0.03301(19) & 0.92675(11) & 0.06259(9) & 2.04(8) \\ \mathrm{C} 21 & -0.13613(21) & 0.89125(11) & 0.01339(10) & 2.60(8) \\ \mathrm{H} 3 & -0.264 & 0.755 & 0.085 & 3.1 \\ \mathrm{H} 4 & -0.340 & 0.627 & 0.058 & 3.7 \\ \mathrm{H} 5 & -0.180 & 0.521 & 0.053 & 4.0 \\ \mathrm{H} 6 & 0.060 & 0.540 & 0.077 & 3.6 \\ \mathrm{H} 8 & 0.255 & 0.655 & 0.116 & 2.8 \\ & & & & \end{array}$




\begin{tabular}{lllll} 
H11a & 0.253 & 0.922 & 0.106 & 3.0 \\
H11b & 0.309 & 0.932 & 0.178 & 3.0 \\
H12 & 0.085 & 0.893 & 0.216 & 2.7 \\
H13 & -0.106 & 0.879 & 0.149 & 2.5 \\
H14a & 0.401 & 0.819 & 0.065 & 3.8 \\
H14b & 0.496 & 0.833 & 0.127 & 3.8 \\
H14c & 0.446 & 0.747 & 0.109 & 3.8 \\
H15a & 0.236 & 0.794 & 0.249 & 3.9 \\
H15b & 0.345 & 0.731 & 0.223 & 3.9 \\
H15c & 0.396 & 0.818 & 0.241 & 3.9 \\
H19a & -0.177 & 1.100 & 0.258 & 4.5 \\
H19b & -0.018 & 1.125 & 0.255 & 4.5 \\
H19c & -0.110 & 1.125 & 0.190 & 4.5 \\
H20a & 0.058 & 0.930 & 0.042 & 2.8 \\
H20b & -0.065 & 0.979 & 0.073 & 2.8 \\
H21a & -0.141 & 0.924 & -0.026 & 3.4 \\
H21b & -0.105 & 0.839 & 0.002 & 3.4 \\
H21c & -0.228 & 0.888 & 0.034 & 3.4 \\
\hline
\end{tabular}

${ }^{a}$ Biso is the Mean of the Principal Axes of the Thermal Ellpsoid.

Table S4. Bond lengths for cis-S7. $^{\text {. }}$.

$\begin{array}{llll}\mathrm{C}(1)-\mathrm{C}(2) & 1.4440(23) & \mathrm{C}(10)-\mathrm{C}(11) & 1.542(3) \\ \mathrm{C}(1)-\mathrm{C}(9) & 1.3682(24) & \mathrm{C}(10)-\mathrm{C}(14) & 1.532(3) \\ \mathrm{C}(1)-\mathrm{C}(13) & 1.5084(24) & \mathrm{C}(10)-\mathrm{C}(15) & 1.539(3) \\ \mathrm{C}(2)-\mathrm{C}(3) & 1.4059(25) & \mathrm{C}(11)-\mathrm{C}(12) & 1.528(3) \\ \mathrm{C}(2)-\mathrm{C}(7) & 1.4152(25) & \mathrm{C}(12)-\mathrm{C}(13) & 1.5555(24) \\ \mathrm{C}(3)-\mathrm{C}(4) & 1.373(3) & \mathrm{C}(12)-\mathrm{C}(16) & 1.5039(25) \\ \mathrm{C}(4)-\mathrm{C}(5) & 1.398(3) & \mathrm{C}(13)-\mathrm{C}(20) & 1.537(3)\end{array}$




$\begin{array}{llll}\mathrm{C}(5)-\mathrm{C}(6) & 1.374(3) & \mathrm{C}(16)-\mathrm{O}(17) & 1.2106(22) \\ \mathrm{C}(6)-\mathrm{C}(7) & 1.394(3) & \mathrm{C}(16)-\mathrm{O}(18) & 1.3348(22) \\ \mathrm{C}(7)-\mathrm{N}(8) & 1.3780(22) & \mathrm{O}(18)-\mathrm{C}(19) & 1.4540(24) \\ \mathrm{N}(8)-\mathrm{C}(9) & 1.3784(22) & \mathrm{C}(20)-\mathrm{C}(21) & 1.516(3) \\ \mathrm{C}(9)-\mathrm{C}(10) & 1.5055(24) & & \end{array}$

Table S5. Bond angles for cis-S7.
$\mathrm{C}(2)-\mathrm{C}(1)-\mathrm{C}(9) \quad 106.86(15) \quad \mathrm{C}(9)-\mathrm{C}(10)-\mathrm{C}(14) \quad 111.80(15)$
$\mathrm{C}(2)-\mathrm{C}(1)-\mathrm{C}(13) \quad 129.69(15) \quad \mathrm{C}(9)-\mathrm{C}(10)-\mathrm{C}(15)$ 108.65(15)
$\mathrm{C}(9)-\mathrm{C}(1)-\mathrm{C}(13) \quad 123.41(15) \quad \mathrm{C}(11)-\mathrm{C}(10)-\mathrm{C}(14)$ 109.22(15)
$\mathrm{C}(1)-\mathrm{C}(2)-\mathrm{C}(3) \quad 135.27(16) \quad \mathrm{C}(11)-\mathrm{C}(10)-\mathrm{C}(15) 110.81(15)$
$\mathrm{C}(1)-\mathrm{C}(2)-\mathrm{C}(7) \quad 106.48(15) \quad \mathrm{C}(14)-\mathrm{C}(10)-\mathrm{C}(15)$ 109.02(15)
$\mathrm{C}(3)-\mathrm{C}(2)-\mathrm{C}(7) \quad 118.23(16) \quad \mathrm{C}(10)-\mathrm{C}(11)-\mathrm{C}(12) 113.41(14)$
$\mathrm{C}(2)-\mathrm{C}(3)-\mathrm{C}(4) \quad 119.24(17) \quad \mathrm{C}(11)-\mathrm{C}(12)-\mathrm{C}(13) 111.80(14)$
$\mathrm{C}(3)-\mathrm{C}(4)-\mathrm{C}(5) \quad 121.33(17) \quad \mathrm{C}(11)-\mathrm{C}(12)-\mathrm{C}(16) 112.07(14)$
$\mathrm{C}(4)-\mathrm{C}(5)-\mathrm{C}(6) \quad 121.27(17) \quad \mathrm{C}(13)-\mathrm{C}(12)-\mathrm{C}(16) 110.86(14)$
$\mathrm{C}(5)-\mathrm{C}(6)-\mathrm{C}(7) \quad 117.65(18) \quad \mathrm{C}(1)-\mathrm{C}(13)-\mathrm{C}(12) \quad 107.30(14)$
$\mathrm{C}(2)-\mathrm{C}(7)-\mathrm{C}(6) \quad 122.26(16) \quad \mathrm{C}(1)-\mathrm{C}(13)-\mathrm{C}(20)$ 113.68(14)
$\mathrm{C}(2)-\mathrm{C}(7)-\mathrm{N}(8) \quad 107.83(15) \quad \mathrm{C}(12)-\mathrm{C}(13)-\mathrm{C}(20) 111.59(14)$
$\mathrm{C}(6)-\mathrm{C}(7)-\mathrm{N}(8) \quad 129.91(17) \quad \mathrm{C}(12)-\mathrm{C}(16)-\mathrm{O}(17)$ 126.08(16)
$\mathrm{C}(7)-\mathrm{N}(8)-\mathrm{C}(9) \quad 108.94(14) \quad \mathrm{C}(12)-\mathrm{C}(16)-\mathrm{O}(18) 111.16(15)$
$\mathrm{C}(1)-\mathrm{C}(9)-\mathrm{N}(8) \quad 109.87(15) \quad \mathrm{O}(17)-\mathrm{C}(16)-\mathrm{O}(18)$ 122.75(17)
$\mathrm{C}(1)-\mathrm{C}(9)-\mathrm{C}(10)$ 127.40(16) $\quad \mathrm{C}(16)-\mathrm{O}(18)-\mathrm{C}(19)$ 116.56(15)
$\mathrm{N}(8)-\mathrm{C}(9)-\mathrm{C}(10) \quad 122.54(15) \quad \mathrm{C}(13)-\mathrm{C}(20)-\mathrm{C}(21)$ 114.69(15)
C(9)-C(10)-C(11) 107.33(1 


\section{References}

1) Smith, A. B. III; Visnick, M.; Haseltine, J. N.; Sprengeler, P. A. Tetrahedron 1986, 42, 2957.

2) Liu, C.; Han, X.; Wang, X.; Widenhoefer, R. J. Am. Chem. Soc. 2004, 126, 3700.

3) Abbiati, G.; Beccalli, E. M.; Broggini, G.; Zoni, C. J. Org. Chem. 2003, 68, 7625.

4) Ferreira, E. M.; Stoltz, B. M. J. Am. Chem. Soc. 2003, 125, 9578.

5) Youn, S. W.; Pastine, S. J.; Sames, D. Org. Lett. 2004, 6, 581.

6) Rodriguez, J. G.; Temprano, F.; Esteban-Calderon, C.; Martinez-Ripoll, M. J. Chem. Soc., Perkin Trans 1 1989, 11, 2117.

7) Marinelli, E. R. Tetrahedron Lett. 1982, 23, 2745. 